hep-th/0405048, UPR-T-1079

\title{
Beyond the veil: Inner horizon instability and holography
}

\author{
Vijay Balasubramanian* and Thomas S. Levit \\ David Rittenhouse Laboratories, University of Pennsylvania, Philadelphia, PA 19104-6396
}

(Dated: September 12, 2018)

\begin{abstract}
We show that scalar perturbations of the eternal, rotating BTZ black hole should lead to an instability of the inner (Cauchy) horizon, preserving strong cosmic censorship. Because of backscattering from the geometry, plane wave modes have a divergent stress tensor at the event horizon, but suitable wavepackets avoid this difficulty, and are dominated at late times by quasinormal behavior. The wavepackets have cuts in the complexified coordinate plane that are controlled by requirements of continuity, single-valuedness and positive energy. Due to a focusing effect, regular wavepackets nevertheless have a divergent stress-energy at the inner horizon, signaling an instability. This instability, which is localized behind the event horizon, is detected holographically as a breakdown in the semiclassical computation of dual CFT expectation values in which the analytic behavior of wavepackets in the complexified coordinate plane plays an integral role. In the dual field theory, this is interpreted as an encoding of physics behind the horizon in the entanglement between otherwise independent CFTs.
\end{abstract}

*Electronic address: vijay@endive.hep.upenn.edu

$\dagger$ Electronic address: tslevi@student.physics.upenn.edu 


\section{Contents}

I. Introduction

II. The rotating BTZ black hole and its embedding 4

A. The geometrv and causal structure 4

$\begin{array}{ll}\text { B. Coordinate systems } & 9\end{array}$

III. The event horizon 10

$\begin{array}{ll}\text { A. The need for wavepackets } & 10\end{array}$

$\begin{array}{ll}\text { B. The invariants } & 13\end{array}$

C. Computation of the wavepackets and their behavior at the event horizon 14

IV. Continuing to the other regions and excursions in the complex plane 15

A. Continuitv of the modes and the wavepacket 15

$\begin{array}{ll}\text { B. The branch cuts and a positive energy condition } & 17\end{array}$

$\begin{array}{ll}\text { V. The Cauchy horizon } & 20\end{array}$

VI. AdS/CFT correlators and detecting the instability 23

A. General considerations 26

B. Computing the 1-point function 29

C. Remarks 33

VII. Conclusions and discussion 36

$\begin{array}{ll}\text { Acknowledgments } & 37\end{array}$

$\begin{array}{ll}\text { References } & 37\end{array}$

\section{INTRODUCTION}

Behind the event horizon of rotating black holes lies an inner horizon which shields a region containing naked timelike singularities. In asymptotically flat space, numerical simulations and analytic studies have shown that a generic, small perturbation can cause such an inner horizon to itself collapse to a singularity [1, 2] (for a more modern study in 
the context of classical gravity see [3]). This is a manifestation of strong cosmic censorship 4]. If such an instability also exists for black holes in AdS space, it presents an opportunity to study how a phenomenon that is entirely localized behind an event horizon is represented holographically in a dual field theory. To this end, we show how a generic scalar perturbation of the rotating BTZ black hole leads to a divergent stress tensor on the inner horizon and explain how the dual CFT would detect the resulting instability.

An important subtlety is that the standard basis of plane wave modes in a BTZ black hole has a divergent stress tensor on the event horizon due to interaction between outgoing and ingoing parts of the solution. This necessitates the construction of wavepackets, which have cuts in the complexified coordinate plane that are controlled by requirements of single-valuedness, continuity and positive energy. The cuts in the complex time plane enable an observer who only integrates over the region outside the black hole, and parts of the complexified coordinate plane, to sense the instability at the inner horizon. From the perspective of the dual CFT this is evidence that entanglement of the two otherwise independent components of the dual field theory encodes the region behind the horizon.

We are familiar with the use of the complexified energy plane as a powerful tool in analyzing scattering amplitudes. For example, a pole at a complex energy indicates a resonance or meta-stable excitation in a system. We are less comfortable with the complexified coordinate plane, but there is increasing evidence that structures in complex time and space contain important information about physics in time dependent backgrounds. In [5, 6, 7, 8, 9] various signatures of physics behind a black hole horizon were associated with the analytic structure of physical quantities in complexified time. In 10] degenerations of the metric of an AdS orbifold in the complex plane of a spatial coordinate were found, surprisingly, to control unitarity and quantization conditions in the real spacetime. A general lesson from all these works and from the present paper might well be that in time-dependent universes, particularly when analytic continuation to a Euclidean section is not possible, structures in the complexfied spacetime are important for physics. 


\section{THE ROTATING BTZ BLACK HOLE AND ITS EMBEDDING}

\section{A. The geometry and causal structure}

The rotating BTZ black hole is the unique rotating black hole solution in three dimensions with a negative cosmological constant. It is locally $A d S_{3}$ and can be described by the metric [1, 12$]$

$$
\begin{aligned}
d s^{2} & =-\frac{\left(r^{2}-r_{+}^{2}\right)\left(r^{2}-r_{-}^{2}\right)}{\Lambda^{2} r^{2}} d t^{2}+\frac{\Lambda^{2} r^{2}}{\left(r^{2}-r_{+}^{2}\right)\left(r^{2}-r_{-}^{2}\right)} d r^{2}+r^{2}\left(d \bar{\phi}-\frac{r_{+} r_{-}}{\Lambda r^{2}} d t\right)^{2}, \\
\bar{\phi} & \sim \bar{\phi}+2 \pi
\end{aligned}
$$

where $r_{ \pm}$are the locations of the outer and inner horizons respectively. The mass and angular momentum are

$$
M=\frac{r_{+}^{2}+r_{-}^{2}}{\Lambda^{2}} \quad ; \quad J=\frac{2 r_{+} r_{-}}{\Lambda} .
$$

It will be easier for discussing the near horizon physics to introduce a different angular coordinate, $\phi_{+}=\bar{\phi}-\Omega_{H} t$, where

$$
\Omega_{H}=\frac{r_{-}}{\Lambda r_{+}}
$$

is the angular velocity of the outer horizon. This coordinate change untwists the $\bar{\phi}$ circle at the outer horizon. The metric in these coordinates becomes

$$
d s^{2}=-\frac{\left(r^{2}-r_{+}^{2}\right)\left(r^{2}-r_{-}^{2}\right)}{\Lambda^{2} r^{2}} d t^{2}+\frac{\Lambda^{2} r^{2}}{\left(r^{2}-r_{+}^{2}\right)\left(r^{2}-r_{-}^{2}\right)} d r^{2}+r^{2}\left(\frac{r_{-}}{\Lambda r_{+} r^{2}}\left(r^{2}-r_{+}^{2}\right) d t+d \phi_{+}\right)^{2} .
$$

We can view this geometry as embedded in $A d S_{3}$ with identifications. $A d S_{3}$ is the hyperboloid $T_{1}^{2}+T_{2}^{2}-X_{1}^{2}-X_{2}^{2}=\Lambda^{2}$ embedded in $R^{2,2}$ with metric $d s^{2}=-d T_{1}^{2}-d T_{2}^{2}+d X_{1}^{2}+d X_{2}^{2}$. One can realize this embedding as

$$
\begin{aligned}
T_{1} & =\sqrt{u} \cosh \left(\frac{r_{+}}{\Lambda} \phi_{+}\right), \\
T_{2} & =\sqrt{u-1} \sinh \left(\kappa_{+} t-\frac{r_{-}}{\Lambda} \phi_{+}\right), \\
X_{1} & =\sqrt{u} \sinh \left(\frac{r_{+}}{\Lambda} \phi_{+}\right), \\
X_{2} & =\sqrt{u-1} \cosh \left(\kappa_{+} t-\frac{r_{-}}{\Lambda} \phi_{+}\right),
\end{aligned}
$$

where

$$
u=\frac{r^{2}-r_{-}^{2}}{r_{+}^{2}-r_{-}^{2}} \quad ; \quad \kappa_{ \pm}=\frac{r_{+}^{2}-r_{-}^{2}}{\Lambda^{2} r_{ \pm}} .
$$

$\kappa_{ \pm}$are the surface gravities at the inner and outer horizon respectively. 
The spacetime can be divided into several regions determined by the norm of the Killing vector $\xi=\partial_{\phi_{+}}$. In region 1, we are outside the black hole's event horizon and $\xi \cdot \xi>r_{+}^{2}$. In region 2, we are between the event horizon and the Cauchy horizon and $r_{+}^{2}>\xi \cdot \xi>r_{-}^{2}$. In region 3 we are behind the Cauchy horizon but outside the singularity, and $r_{-}^{2}>\xi \cdot \xi>0$. Finally, before making the identification on $\phi_{+}$, a region where the norm is less than zero exists (this would be region 4). Upon making the identification on $\phi_{+}$this region would contain closed timelike curves. We cutoff the spacetime at the surface $\xi \cdot \xi=0$, treating it as a singularity, so the spacetime has no closed timelike curves. This singularity is timelike if $r_{-} \neq 0$ (i.e. the black hole has non-vanishing angular momentum) and spacelike if $r_{-}=0$. We will principally be interested in the finite angular momentum case.

In terms of the embedding coordinates, $T_{1}^{2}-X_{1}^{2}=u$ and $T_{2}^{2}-X_{2}^{2}=1-u$, so that the regions can also be distinguished by

$$
\begin{array}{rll}
\text { region 1: } & T_{1}^{2}-X_{1}^{2} \geq 0, & T_{2}^{2}-X_{2}^{2} \leq 0 \\
\text { region 2: } & T_{1}^{2}-X_{1}^{2} \geq 0, & T_{2}^{2}-X_{2}^{2} \geq 0 \\
\text { regions 3\& 4: } & T_{1}^{2}-X_{1}^{2} \leq 0, & T_{2}^{2}-X_{2}^{2} \geq 0 .
\end{array}
$$

Each of these regions describes several disconnected components of the maximally extended geometry which can be distinguished by the signs of certain combinations of the embedding coordinates. Following the procedure of [5, 6, 7], denote the different regions by $A_{\eta_{1} \eta_{2}}$, where $A=1, \ldots, 3$ and $\eta_{1}, \eta_{2}= \pm$ are the signs of the two combinations $T_{1}+X_{1}$ and $T_{2}+X_{2}$. The boundary of the spacetime is given by two disconnected cylinders. Fig. [1 is a Penrose diagram.

A null geodesic starting from a location outside the black hole takes infinite BTZ coordinate time to reach either the past or future event horizon where the coordinate system breaks down. The future (past) event horizon is line that is approached as $r \rightarrow r_{+}$while $t \rightarrow \infty(t \rightarrow-\infty)$. The intersection of the past and future event horizons occurs in the limit $r \rightarrow r_{+}$for any finite $t$. (We will define a coordinate system that makes this apparent later.) In a similar way, one finds the right (left) Cauchy horizon in the $2_{++}$region is given by $r \rightarrow r_{-}$and $t \rightarrow+\infty(t \rightarrow-\infty)$. Fig. 2 shows the horizon structure in the $1_{++}$and $2_{++}$ regions.

Beyond the inner horizon at $r_{-}$, the timelike singularity requires a specification of boundary conditions that leads to a breakdown in predictability. In asymptotically flat space, a 


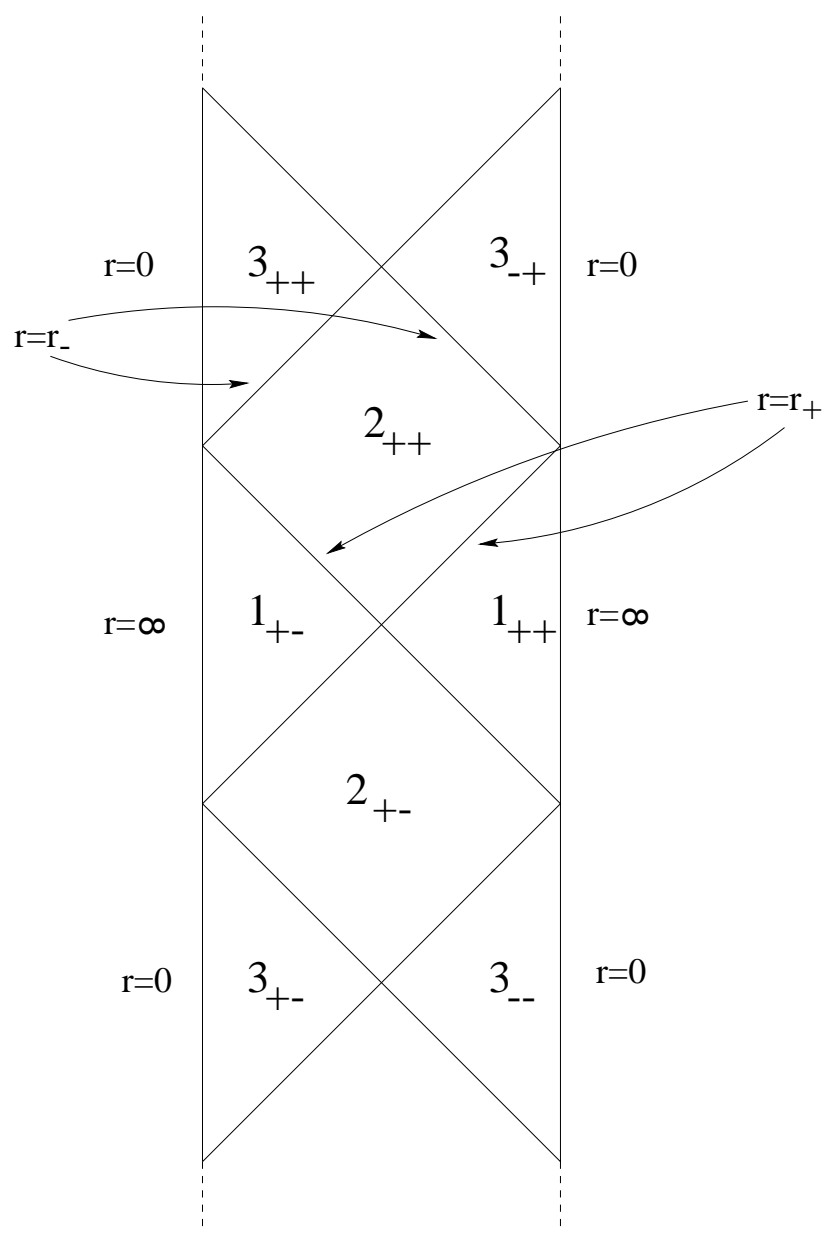

FIG. 1: Penrose diagram detailing the causal structure of the rotating BTZ black hole [12]. The diagram repeats itself indefinitely above and below. The vertical boundaries of the regions $3_{ \pm \pm}$are singularities while the vertical boundaries of the regions $1_{ \pm \pm}$are asymptotically AdS boundaries. We study an instability of the inner horizon at the locations marked $r_{-}$that results from the gravitational focusing of wavepackets.

dynamical instability of the inner horizon of rotating and charged black holes is triggered by generic small perturbations, cutting off the region behind it and supporting the strong cosmic censorship conjecture 1, 2]. Numerical and analytic studies of shells of matter in BTZ black holes suggest an instability at the inner horizon [13]. We will show that a generic scalar perturbation has divergent stress-energy on the inner horizon which signals that the inner horizon is unstable to scalar perturbations.

- Excursions in the complex plane: Since we expect something to break down at the 


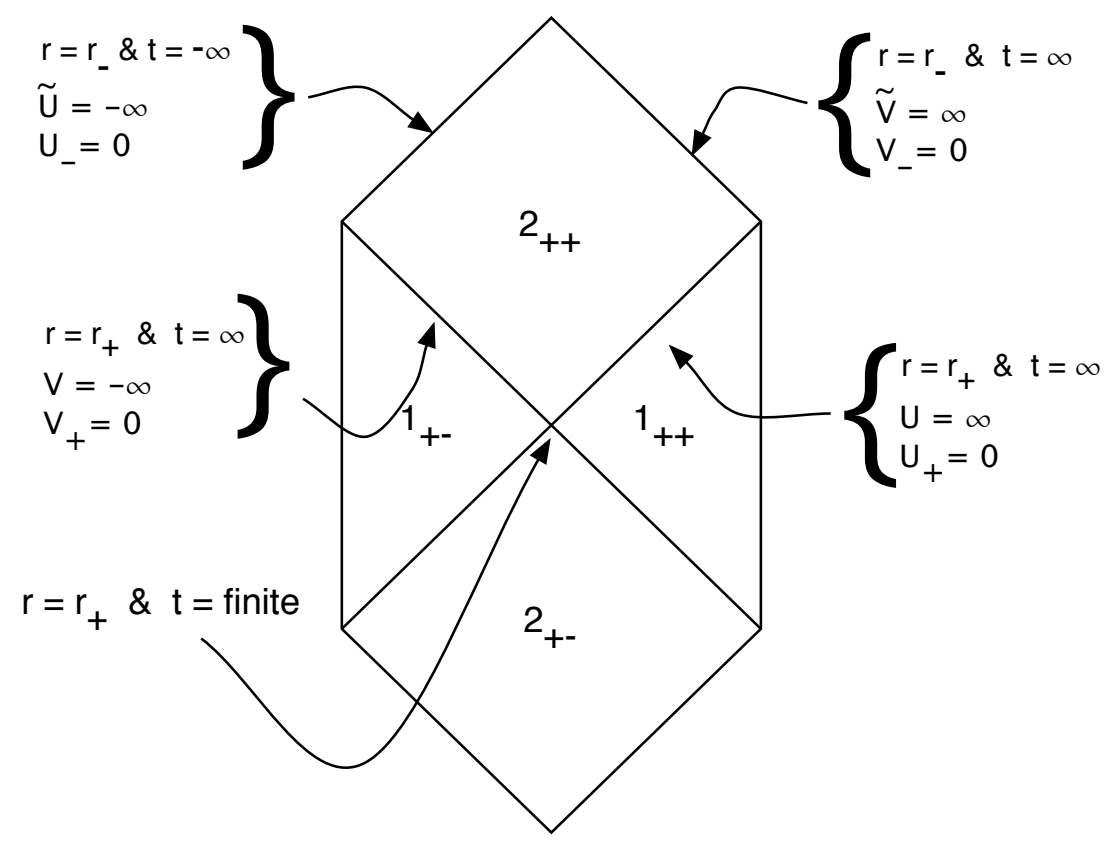

FIG. 2: The Penrose diagram for regions of interest, detailing some of the horizon structure in the various coordinate systems. The horizons in the other regions are similar.

Cauchy horizon, we will principally be interested in coordinate patches for a set of regions that do not cross it. We focus on the regions $1_{+ \pm}$and $2_{+ \pm}$where

$$
\begin{aligned}
& T_{1}+X_{1}=\sqrt{u} \exp \left(\frac{r_{+}}{\Lambda} \phi_{+}\right), \\
& T_{2}+X_{2}=\sqrt{u-1} \exp \left(\kappa_{+} t-\frac{r_{-}}{\Lambda} \phi_{+}\right),
\end{aligned}
$$

and $T_{1}+X_{1}$ is always positive. We can now move from region to region by taking excursions in the complexified BTZ coordinate plane. In region $1_{++}$(the right asymptotic region), $u>1$ and $t$ is real. To move to $2_{++}$we first take $0<u<1$. The square root in the embedding equation (2.9) introduces a factor of $i$, so to keep the embedding real, and the sign of $T_{2}+X_{2}$ positive, we must take $t \rightarrow t-i \beta_{*} / 4$, where $\beta_{*}=2 \pi / \kappa_{+}$is the inverse Hawking temperature of the black hole. We have selected a specific branch for continuing $t$, but there is nothing in the embedding equations that prevents us from taking a shift of $+3 i \beta_{*} / 4$ or any other shift separated by a factor of $\beta_{*}$. Later on, we will see that dynamical issues will reduce this freedom to a single choice of branch. Since the shift in the complex direction is always a constant, and the $t$ dependence in the metric is always in the form of a $d t$ term, it does not affect the metric. 
As is well known, BTZ coordinates (2.4) nominally break down at the outer horizon of the black hole and require some sort of continuation to cover the spacetime. If we are only interested in physics within one of the regions $A_{ \pm \pm}$we could define separate BTZ-like coordinate patches in each region, defined by equations like (2.8, 2.9). This amounts to naively setting $r<r_{+}$in (2.4). However, if we start in one region and attempt to move into another while maintaining the embedding equations (2.8, 2.9), the different regions appear as different sections of a complexified BTZ coordinate plane each having a real metric. This perspective has been used in BTZ and other backgrounds [5, 6, 7, 8, 10]. Examining the relation between BTZ and Kruskal coordinates for the black hole provides further insight into how this works. It is not possible to find a coordinate system that covers both the outer and inner horizon. One set of Kruskal coordinates, which extends from the AdS boundary up to, but not including the Cauchy horizon, is related to the BTZ coordinates (2.4) by [12]

$$
\begin{array}{r}
2_{++}: \quad r_{-}<r \leq r_{+}\left\{\begin{array}{l}
X_{+}=\left[\left(\frac{r_{+}-r}{r+r_{+}}\right)\left(\frac{r+r_{-}}{r-r_{-}}\right)^{r_{-} / r_{+}}\right]^{1 / 2} \sinh \kappa_{+} t \\
T_{+}=\left[\left(\frac{r+-r}{r+r_{+}}\right)\left(\frac{r+r_{-}}{r-r_{-}}\right)^{r_{-} / r_{+}}\right]^{1 / 2} \cosh \kappa_{+} t
\end{array}\right. \\
1_{++}: \quad r_{+} \leq r<\infty\left\{\begin{array}{l}
X_{+}=\left[\left(\frac{r-r_{+}}{r+r_{+}}\right)\left(\frac{r+r_{-}}{r-r_{-}}\right)^{r_{-} / r_{+}}\right]^{1 / 2} \cosh \kappa_{+} t \\
T_{+}=\left[\left(\frac{r-r_{+}}{r+r_{+}}\right)\left(\frac{r+r_{-}}{r-r_{-}}\right)^{r_{-} / r_{+}}\right]^{1 / 2} \sinh \kappa_{+} t
\end{array}\right.
\end{array}
$$

The future and past event horizons occur at $X_{+}= \pm T_{+}$. This would usually be described as a choice of different BTZ coordinate patches for each region. Here, we instead interpret passing through the horizon as a shift in $t$ by $-i \beta_{*} / 4$, which exchanges the sinh and cosh in (2.10) and (2.11).

In a similar way, to reach $1_{+-}$from $1_{++}$we take $t \rightarrow t-i \beta_{*} / 2$, and to reach $2_{+-}$we take $t \rightarrow t-3 i \beta_{*} / 4$. From (2.9) we can describe $1_{++}$using either $t$ or $t+i n \beta_{*}$, where $n$ is an integer. A similar formula can be found for the other regions. Later on, we will see that this freedom will be reduced to a single choice of branch.

The Euclidean continuation of this spacetime is achieved by taking $t=-i \tau$ and continuing $r_{-}=-i \tilde{r}_{-}$to keep the metric real:

$$
d s_{E}^{2}=\frac{\left(r^{2}-r_{+}^{2}\right)\left(r^{2}+\tilde{r}_{-}^{2}\right)}{\Lambda^{2} r^{2}} d \tau^{2}+\frac{\Lambda^{2} r^{2}}{\left(r^{2}-r_{+}^{2}\right)\left(r^{2}+\tilde{r}_{-}^{2}\right)} d r^{2}+r^{2}\left(d \phi_{+}-\frac{\tilde{r}_{-}}{\Lambda r_{+} r^{2}}\left(r^{2}-r_{+}^{2}\right) d \tau\right)^{2} .
$$

The range of $r$ is now $r_{+}<r<\infty$ and to avoid a conical singularity at $r=r_{+}, \tau$ must be periodic with period $\beta_{*}$. Intriguingly, a discrete motion by $\tau=-\beta_{*} / 2$ in the complexified 
BTZ time in effect takes us between disconnected components of the Lorentzian spacetime boundary.

\section{B. Coordinate systems}

Throughout the paper we will switch between various appropriate coordinate systems. For reference we define them here and where appropriate give their values in the different regions of interest. We have already defined the BTZ coordinates. Tortoise coordinates in region $1_{++}$are given by

$$
r_{*}=\frac{1}{2 \kappa_{+}} \ln (u-1)-\frac{1}{2 \kappa_{-}} \ln u \quad ; \quad U=t-r_{*} \quad ; \quad V=t+r_{*} .
$$

In region $1_{++}$, the location of the past (future) event horizon in these coordinates is $V=-\infty$ $(U=\infty)$. In region $2_{++}, r_{*}$ acquires an imaginary part by definition, and $t$ shifts into the complex plane as described above. It will be useful later to isolate the real parts of these coordinates so we define

$$
\tilde{r}_{*}=\frac{1}{2 \kappa_{+}} \ln (1-u)-\frac{1}{2 \kappa_{-}} \ln u \quad ; \quad \tilde{U}=t-\tilde{r}_{*} \quad ; \quad \tilde{V}=t+\tilde{r}_{*}
$$

Here $\tilde{r}$ is the real part of $r_{*}$ and $t$ does not include the finite shift of $-i \beta_{*} / 4$ in region $2_{++}$. In these coordinates, the left (right) Cauchy horizon is $\tilde{U}=-\infty(\tilde{V}=\infty)$.

As described in [12] there are two sets of Kruskal coordinates, one covering the outer horizon but not the inner horizon, and vice versa. The outer coordinates are given by (2.10, 2.11). From these, we can construct null Kruskal coordinates

$$
U_{+}=X_{+}-T_{+} \quad ; \quad V_{+}=X_{+}+T_{+}
$$

These coordinates break down at the inner horizon as they diverge and the metric has vanishing determinant. In terms of these coordinates we have

$$
\begin{array}{cl}
1_{++}: V_{+}, U_{+}>0 & 1_{+-}: V_{+}, U_{+}<0 \\
2_{++}: U_{+}<0, V_{+}>0 & 2_{+-}: V_{+}<0, U_{+}>0
\end{array}
$$

and the past and future event horizons occur at $V_{+}=0$ and $U_{+}=0$ respectively.

Kruskal coordinates that cover the inner horizon (but not the outer horizon) can also be defined and their relation with BTZ coordinates in the region $2_{++}, r_{-} \leq r<r_{+}$are given 
by

$$
\begin{gathered}
X_{-}=-\left[\left(\frac{r-r_{-}}{r+r_{-}}\right)\left(\frac{r_{+}+r}{r_{+}-r}\right)^{r_{+} / r_{-}}\right]^{1 / 2} \sinh \left(\kappa_{-} t\right) \\
T_{-}=\left[\left(\frac{r-r_{-}}{r+r_{-}}\right)\left(\frac{r_{+}+r}{r_{+}-r}\right)^{r_{+} / r_{-}}\right]^{1 / 2} \cosh \left(\kappa_{-} t\right), \\
\phi_{-}=\bar{\phi}-\Omega_{C} t,
\end{gathered}
$$

where

$$
\Omega_{C}=\frac{r_{+}}{r_{-} \Lambda}
$$

is the angular velocity of the inner horizon. The angular coordinate $\phi$ has be redefined to obtain well-defined metric components at the inner horizon. From these, we can construct null Kruskal coordinates

$$
U_{-}=X_{-}-T_{-} \quad ; \quad V_{-}=X_{-}+T_{-}
$$

The left (right) Cauchy horizon in region $2_{++}$is the line $U_{-}=X_{-}-T_{-}=0\left(V_{-}=X_{-}+T_{-}=\right.$ $0)$.

\section{THE EVENT HORIZON}

The first goal of this paper is to demonstrate that the Cauchy horizon of the rotating BTZ black hole is unstable to small scalar perturbations. To show this we construct classical scalar wavepackets, the stress tensor invariants of which are finite at the outer horizon, but nevertheless diverge due to focusing effects at the inner horizon. In this section we show that that standard basis of plane wave normalizable modes in the asymptotic region $1_{++}$is singular at the event horizon. The divergence arises from an interaction between the ingoing and outgoing pieces of the mode. We then show how to construct wavepackets with regular behaviour at the outer horizon. These wavepackets can then be used to diagnose potential blueshifting instabilities at the inner horizon.

\section{A. The need for wavepackets}

The plane-wave normalizable modes in region $1_{++}$are [14]

$$
\phi_{n}(x)=e^{-i \omega t+i n \phi_{+}+i \Omega_{H} n t}(u-1)^{\alpha} u^{-h_{+}-\alpha} F\left(\alpha+\beta+h_{+}, \alpha-\beta+h_{+} ; 2 h_{+} ; u^{-1}\right),
$$


where $F$ is a hypergeometric function,

$$
\begin{aligned}
\alpha & =\frac{i \Lambda^{2}}{2\left(r_{+}^{2}-r_{-}^{2}\right)}\left(r_{+} \omega-r_{-} n / \Lambda\right) \\
\beta & =\frac{i \Lambda^{2}}{2\left(r_{+}^{2}-r_{-}^{2}\right)}\left(r_{-} \omega-r_{+} n / \Lambda\right), \\
h_{ \pm} & =\frac{1}{2}(1 \pm \nu)=\frac{1}{2}\left(1 \pm \sqrt{1+m^{2}}\right),
\end{aligned}
$$

and $m$ is the mass of the scalar field. This representation of the hypergeometric function is well adapted to describe the behavior on the boundary, and one can readily verify that $\phi_{n}$ decays as $r^{-2 h_{+}}$there. To examine the behavior near the event horizon we use the linear transformations of the hypergeometric function to obtain [15]

$$
\begin{array}{r}
\phi_{n}(x)=e^{-i \omega t+i n \phi_{+}+i \Omega_{H} n t}\left[A(u-1)^{\alpha} u^{\beta} F\left(\alpha+\beta+h_{+}, \alpha+\beta+h_{-} ; 2 \alpha+1 ; 1-u\right)+\right. \\
\left.B(u-1)^{-\alpha} u^{-\beta} F\left(-\alpha-\beta+h_{+},-\alpha-\beta+h_{-} ;-2 \alpha+1 ; 1-u\right)\right]
\end{array}
$$

where

$$
A=\frac{\Gamma\left(2 h_{+}\right) \Gamma(-2 \alpha)}{\Gamma\left(-\alpha-\beta+h_{+}\right) \Gamma\left(-\alpha+\beta+h_{+}\right)} \quad ; \quad B=\frac{\Gamma\left(2 h_{+}\right) \Gamma(2 \alpha)}{\Gamma\left(\alpha+\beta+h_{+}\right) \Gamma\left(\alpha-\beta+h_{+}\right)} .
$$

The coefficient $A$ multiplies the outgoing part of the mode while $B$ multiplies the ingoing part. Any normalizable mode solution of the wave equation in the BTZ background will have both outgoing and ingoing pieces because of backscattering from the geometry. By contrast in asymptotically flat space we could have chosen a purely ingoing normalizable solution.

Near the event horizon $(u=1)$, the leading terms in the solution are

$$
\phi_{n}(x) \approx e^{-i \omega t+i n \phi_{+}+i \Omega_{H} n t}\left[A(u-1)^{\alpha}+B(u-1)^{-\alpha}\right]
$$

An invariant for checking the behavior of the field at any point is the trace of its stress-energy tensor. The stress-energy tensor for a minimally coupled scalar field is [16]

$$
T_{\mu \nu}=\frac{1}{2}\left(\partial_{\mu} \phi \partial_{\nu} \phi^{*}+\partial_{\nu} \phi \partial_{\mu} \phi^{*}\right)-\frac{1}{2} g_{\mu \nu}\left(g^{\rho \sigma} \partial_{\rho} \phi \partial_{\sigma} \phi^{*}+m^{2}|\phi|^{2}\right) .
$$

Taking the trace we obtain (for three spacetime dimensions)

$$
T_{\mu}^{\mu}=-\frac{1}{2} g^{\mu \nu} \partial_{\mu} \phi \partial_{\nu} \phi^{*}-\frac{3}{2} m^{2}|\phi|^{2} .
$$


$\phi_{n}$ is well behaved on the event horizon, so any divergent behavior will come from the first part of the trace (note that this is also of the same form as the kinetic term in the Lagrangian). Using our mode solution (3.5) for real $\omega$ we find

$$
\begin{aligned}
g^{\mu \nu} \partial_{\mu} \phi_{n} \partial_{\nu} \phi_{n}^{*} & \approx-2\left[A B^{*}(u-1)^{2 \alpha}+B A^{*}(u-1)^{-2 \alpha}\right] \frac{\Lambda^{2}}{\left(r_{+}^{2}-r_{-}^{2}\right)\left(r^{2}-r_{+}^{2}\right)}\left(r_{+} \omega-r_{-} n / \Lambda\right)^{2} \\
& +\frac{n^{2}}{r_{+}^{2}-r_{-}^{2}}\left|\phi_{n}\right|^{2} .
\end{aligned}
$$

Since this expression is independent of $t$, the stress tensor diverges everywhere along the event horizon, which is reached by by taking $r \rightarrow r_{+}$while $t \rightarrow \pm \infty$.

Notice that the divergent terms depend on a product of $A$ and $B$ and hence involve an interaction between the outgoing and ingoing parts of the solution. This is the classic phenomenon of large center of mass energies developing in in-out collisions close to a black hole horizon. In asymptotically flat space, it is possible to choose purely ingoing boundary conditions at the event horizon but the the AdS geometry does not allow this for normalizable solutions. Purely ingoing modes will either be non-normalizable in the sense of diverging at the AdS boundary or will involve the complex frequencies. The latter solutions, the quasi-normal modes, are determined by setting the outgoing coefficient $A$ in (3.4) to zero. (See 17] for other studies of quasinormal modes in AdS black holes.) This is achieved by choosing the complex frequencies

$$
\begin{aligned}
& \omega_{L}=-2 i\left(h_{+}+k\right)\left(r_{+}-r_{-}\right) / \Lambda^{2}+n / \Lambda, \\
& \omega_{R}=-2 i\left(h_{+}+k\right)\left(r_{+}+r_{-}\right) / \Lambda^{2}-n / \Lambda,
\end{aligned}
$$

where $k=0,1 \ldots$ and $n$ is the angular frequency in the mode (3.3). Mode solutions with these frequencies decay exponentially in the future, reflecting the infall into the horizon, but they grow exponentially in the past. Due to this behavior we find a finite trace for the stress tensor on the future horizon but a divergence on the past horizon. Therefore, the quasinormal modes do not form a suitable set of perturbations that are regular in the black hole exterior. Below we will construct wavepackets with the necessary regularity and show that they are dominated at late times by quasinormal behavior. 


\section{B. The invariants}

We have not yet fixed any normalization on the modes so we are free to multiply by an overall constant. For ease of computations later we divide the solutions by an overall factor of $A$, giving

$$
\phi_{n}(x) \approx e^{-i \omega t+i n \phi_{+}+i \Omega_{H} n t}\left[(u-1)^{\alpha}+\frac{B(\omega)}{A(\omega)}(u-1)^{-\alpha}\right],
$$

for the near horizon behavior. One can view the ratio $B / A$ as the dispersion for the ingoing wave as a result of scattering from the gravitational well of AdS. From (2.13), near the outer horizon $r_{*} \approx\left(1 / 2 \kappa_{+}\right) \ln (u-1)$. The mode solution near the horizon can be approximated as

$$
\phi_{n}(x) \approx e^{i n \phi_{+}}\left(e^{-i \omega U} e^{i \Omega_{H} n U}+\frac{B(\omega)}{A(\omega)} e^{-i \omega V} e^{i \Omega_{H} n V}\right),
$$

where $\Omega_{H}=\frac{r_{-}}{r_{+} \Lambda}$ is the angular velocity of the outer horizon and we are using the tortoise coordinates defined in (2.13) . To form a wavepacket we sum over modes of different $\omega$ with some weighting kernel $H(w)$. Define the functions

$$
\begin{gathered}
f(U)=\int_{-\infty}^{\infty} d \omega e^{-i \omega U} H(\omega), \\
g(V)=\int_{-\infty}^{\infty} d \omega e^{-i \omega V} \frac{B(\omega)}{A(\omega)} H(\omega) .
\end{gathered}
$$

Near the event horizon, the wavepacket is

$$
\phi_{w p}(x) \approx e^{i n \phi_{+}}\left[f(U) e^{i \Omega_{H} n U}+g(V) e^{i \Omega_{H} n V}\right]
$$

Here $f(U)$ is the outgoing part of the wavepacket and $g(V)$ is the ingoing part. We need $f$ to be small at the future horizon and $g$ to be small at the past horizon in order to have a finite stress-energy. We wish to compute the trace of the stress-energy tensor with our wavepacket. As before, any divergences come from the kinetic term. Setting $f^{\prime}=d f / d U$ and $g^{\prime}=d g / d V$, we obtain

$$
\begin{aligned}
g^{\mu \nu} \partial_{\mu} \phi_{w p} \partial_{\nu} \phi_{w p}^{*} \approx \frac{2 \Lambda^{2} r_{+}^{2}}{\left(r_{+}^{2}-r_{-}^{2}\right)^{2}} e^{\kappa_{+}(U-V)} & \left(e^{i \Omega_{H} n(U-V)}\left[-f^{\prime} g^{\prime *}+i \Omega_{H} n f^{\prime} g^{*}-i \Omega_{H} n f g^{\prime *}-\Omega_{H}^{2} n^{2} f g^{*}\right]\right. \\
& + \text { c.c. })+\frac{n^{2}}{r_{+}^{2}-r_{-}^{2}}\left|\phi_{w p}\right|^{2}
\end{aligned}
$$

Despite the apparent divergence as $e^{\kappa_{+} U}\left(e^{-\kappa_{+} V}\right)$ as we approach the future (past) event horizon, this stress tensor trace can be in fact be finite if the kernel $H(\omega)$ in (3.13, 3.14) is appropriately chosen. We turn to this below. 


\section{Computation of the wavepackets and their behavior at the event horizon}

To get a finite stress tensor at the event horizon the kernel $H(\omega)$ (3.13, 3.14) must be chosen to suppress the $e^{\kappa_{+}(U-V)}$ that appears as an overall factor in (3.16) $)$. We will not try to find a general basis of wavepackets satisfying this condition, but will instead give sufficient conditions on $H(\omega)$ that lead to regular wavepackets.

Let us assume that $H(\omega)$ is a meromorphic function of $\omega$ which falls off sufficiently quickly at infinity to allow use of contour integration in (3.13, 3.14). The potential divergence at the past event horizon $(V \rightarrow-\infty)$ in (3.16) occurs because of the overall factor of $e^{-\kappa_{+} V}$. To suppress this, $g(V)$ must decay sufficiently quickly as $V \rightarrow-\infty$. (Similarly $f(U)$ must decay sufficiently quickly as $U \rightarrow \infty$ for the stress tensor to be finite on the future horizon.) To consider the behavior of $g(V)$ on the past horizon, we can compute the integral (3.14) by closing the contour in the upper half plane (UHP) for convergence on the semicircle at infinity. Poles can arise from the the factor $B / A$ and from the kernel $H$. From (3.4) we have

$$
\frac{B(\omega)}{A(\omega)}=\frac{\Gamma(2 \alpha) \Gamma\left(h_{+}-\alpha-\beta\right) \Gamma\left(h_{+}-\alpha+\beta\right)}{\Gamma(-2 \alpha) \Gamma\left(h_{+}+\alpha+\beta\right) \Gamma\left(h_{+}+\alpha-\beta\right)} .
$$

The term $\Gamma(2 \alpha)$ in the numerator gives rise to poles at

$$
\omega=i k \kappa_{+}+\frac{r_{-}}{r_{+}} n
$$

where $k=1,2, \ldots$ (the $k=0$ pole does not contribute because the $\Gamma(-2 \alpha)$ in the denominator cancels it). These poles are in the upper half plane and contribute. (The other Gamma functions in the numerator give rise to poles in the lower half plane (LHP) and do not contribute.) The dominant contribution will be from the $k=1$ pole as $V \rightarrow-\infty$ which leads to a behavior of $g(V) \sim e^{\kappa_{+} V}$. This precisely cancels the overall divergent factor $e^{-\kappa_{+} V}$ in (3.16) that appears at the past horizon $V \rightarrow-\infty$. Therefore, to have a finite stress tensor trace on the past horizon it suffices to require that any poles in $H$ that lie in the UHP have $\operatorname{Im}(\omega) \geq \kappa_{+}$. A similar analysis on the future horizon for $f(U)$ leads to the condition that any poles in $H$ that lie in the LHP have $\operatorname{Im}(\omega) \leq-\kappa_{+}$. In summary, for the stress tensor to have a finite trace it is sufficient that poles in $H(\omega)$ satisfy the condition

$$
|\operatorname{Im}(\omega)| \geq \kappa_{+} .
$$

(For simplicity we have assumed that $H(\omega)$ does not cancel any poles in $B / A$, and that there are no double poles. These situations can be handled in a straightforward manner.) 
Other invariants constructed out of the stress tensor are also expected to be finite under these conditions.

We can also show that on the future horizon $(U \rightarrow \infty)$ the late time behavior $(V \rightarrow \infty)$ of the regular wavepackets is dominated by quasinormal modes. The outgoing part of the wavepacket, which is controlled by $f(U)$, is exponentially suppressed by construction; so we are interested in the behavior of the ingoing part, $g(V)$. To compute the late time behavior of this part, i.e., as $V \rightarrow \infty$ in (13.14), we close the contour in the LHP. Then, in addition to any poles in $H(\omega)$ we pick up the poles from the latter two gamma functions in the numerator of (3.17). The poles in $\Gamma\left(h_{+}-\alpha-\beta\right)$ occur at

$$
\omega=\omega_{L}=-2 i\left(h_{+}+k\right)\left(r_{+}-r_{-}\right) / \Lambda^{2}+n / \Lambda \quad ; \quad k=0,1, \ldots,
$$

The poles in $\Gamma\left(h_{+}-\alpha+\beta\right)$ occur at

$$
\omega=\omega_{R}=-2 i\left(h_{+}+k\right)\left(r_{+}+r_{-}\right) / \Lambda^{2}-n / \Lambda \quad ; \quad k=0,1, \ldots
$$

¿From the residues at these poles we see that at late times on the future horizon, the wavepacket is dominated by a sum of quasinormal modes as we should expect.

\section{CONTINUING TO THE OTHER REGIONS AND EXCURSIONS IN THE COMPLEX PLANE}

In the previous section we found conditions on wavepackets in the $1_{++}$asymptotic region which led to a well-defined stress tensor on the outer horizon. In order to study whether such a packet can seed an instability of the inner horizon we must continue the solution to other regions of the black hole. Requiring single valuedness, continuity and positive energy places conditions on both the kernel that determines the wavepacket and the locations of branch cuts in the complex Kruskal coordinate plane. Below we will determine these conditions and translate them back to BTZ coordinates.

\section{A. Continuity of the modes and the wavepacket}

First we examine continuity of the packets across the event horizon. Near the event horizon in the region $1_{++}$the relation between BTZ and outer Kruskal coordinates (2.15) is

$$
t=\frac{1}{2 \kappa_{+}}\left(\ln V_{+}-\ln U_{+}\right) \quad ; \quad u-1 \approx \frac{4 r_{+}^{2}}{r_{+}^{2}-r_{-}^{2}}\left(\frac{r_{+}-r_{-}}{r_{+}+r_{-}}\right)^{r_{-} / r_{+}} U_{+} V_{+} .
$$


Using these transformations the null tortoise coordinates (2.13) are

$$
U=-\frac{1}{\kappa_{+}}\left[\ln \gamma+\ln U_{+}\right] \quad ; \quad V=\frac{1}{\kappa_{+}}\left[\ln \gamma+\ln V_{+}\right],
$$

where

$$
\gamma^{2}=\frac{4 r_{+}^{2}}{r_{+}^{2}-r_{-}^{2}}\left(\frac{r_{+}-r_{-}}{r_{+}+r_{-}}\right)^{r_{-} / r_{+}}
$$

We can find the wavepacket in the other regions all the way up to the inner horizon by letting the Kruskal coordinates range over all real values from $-\infty$ to $\infty$. First, consider the modes (3.12) as we pass into either region 2 from the asymptotic region $1_{++}$. As we pass through the future horizon at $U_{+}=0$, the outgoing ( $U$ dependent) part of the solution is

$$
e^{-i \omega U+i \Omega_{H} n U} \sim e^{-i / \kappa_{+} \omega \ln U_{+}+i / \kappa_{+} \Omega_{H} n \ln U_{+}} .
$$

This is discontinuous across $U_{+}=0$ because of the branch cut in the log in the exponent. Similarly, the ingoing ( $V$ dependent) part of the mode is discontinuous across the the past horizon $\left(V_{+}=0\right)$. We have already shown that the stress tensor for the mode solutions diverges on the event horizon precisely because the outgoing (ingoing) part is non-vanishing on the future (past) horizon. The latter problem was cured by making wavepackets in which the outgoing (ingoing) part vanished on the future (past) horizon. We will see below that continuity of the wavepackets is linked to the finiteness and continuity of the stress tensor.

Near the event horizon the wavepacket is given by (3.15). We examine the outgoing $(f(U))$ and ingoing $(g(V))$ parts separately. Near the future event horizon $\left(U_{+}=0\right)$ the ingoing part of the wavepacket is manifestly continuous and differentiable. The dangerous part is the outgoing piece $f(U)$. The contour integral defining $f(U)$ (3.13) receives contributions from poles in the kernel $H(\omega)$ that lie in the LHP. We parametrize the location of these poles as $\omega=-a i \kappa_{+} \mp b \kappa_{+}$where $a, b$ are positive, real constants. Then, near the future horizon, the outgoing part of the wavepacket behaves as

$$
e^{i \Omega_{H} n U} f(U) \sim e^{i\left(\Omega_{H} n \pm b \kappa_{+}\right) U-a \kappa_{+} U} \sim \exp \left[-i\left(\frac{\Omega_{H} n}{\kappa_{+}} \pm b\right)\left(\ln \gamma+\ln U_{+}\right)+a\left(\ln U_{+}+\ln \gamma\right)\right] .
$$

When $U_{+}<0$ the right hand side of this equation becomes

$$
\exp \left[\left(\frac{\Omega_{H} n}{\kappa_{+}} \pm b\right) m \pi+i a m \pi\right] \exp \left[-i\left(\frac{\Omega_{H} n}{\kappa_{+}} \pm b\right)\left(\ln \gamma+\ln \left(-U_{+}\right)\right)+a\left(\ln \left(-U_{+}\right)+\ln \gamma\right)\right],
$$

where $m$ represents the choice of branch in the log, which we will come back to shortly. The second exponential is the original wavepacket again. Therefore, if $a>0$, the expression vanishes at $U_{+}=0$ and the wavepacket is continuous. If $a \geq 1$ then the packet is differentiable, 
and so on. Similar issues of continuity and differentiability of wavepackets arise in studies of standard Schwarzschild black holes [18, 19].

Similarly, at the past horizon $\left(V_{+}=0\right)$ the outgoing part of the wavepacket is manifestly continuous and differentiable, but the ingoing piece $(g(V))$ requires analysis. The contour integral defining $g(V)$ (3.14) near the past horizon receives contributions from the poles in both $B(\omega) / A(\omega)$ and the kernel $H(\omega)$ that lie in the upper half plane. The poles from $B / A$ (3.18) lead to

$$
e^{i \Omega_{H} n V} g(V) \sim \sum_{k=1}^{\infty} \operatorname{Res}_{\omega_{k}}(H B / A) e^{k \kappa_{+} V} \sim \sum_{k=1}^{\infty} \operatorname{Res}_{\omega_{k}}(H B / A) \gamma^{k} V_{+}^{k},
$$

as $V_{+} \rightarrow 0$. Since $k$ are positive integers these terms are $C^{\infty}$ at $V_{+}=0$. Then, following the analysis above, one finds similar requirements for any poles of $H(\omega)$ in the UHP and a branch cut from $\ln V_{+}$.

One can check that the trace of the stress tensor is continuous so long as $H(\omega)$ only has poles at $\omega_{p}$ with $\left|\operatorname{Im}\left(\omega_{p}\right)\right|>\kappa_{+}$. The requirement that the stress tensor is continuous is thus sufficient to ensure that the stress tensor is finite and that the wavepacket is continuous at the event horizon.

\section{B. The branch cuts and a positive energy condition}

As discussed above, the regular wavepackets have branch cuts in both the coordinates $U_{+}$ and $V_{+}$that start at the origin and extend out to infinity. We would like a physical criterion for placing the cuts in the complex plane. Related issues arise in studies of Schwarzschild black holes in asymptotically flat space and even in the Rindler description of Minkowski space. In these cases, a method for determining the placement of the branch cuts was given by Unruh [18]. Here we extend this procedure to the BTZ spacetime. Essentially, the method involves using a condition of positive energy to select whether the cuts extend from the origin into the upper or lower half Kruskal coordinate planes. This is a discrete choice that does not depend on the angular momentum in the solution. For ease of computation, therefore, we will determine the location of the cuts in the non-rotating case with $r_{-}=0$. The result will apply also to the rotating case.

Unruh's method relies on finding null vectors which become of the Killing type on the past and future event horizons. In Kruskal coordinates, the non-rotating metric can be 
written [12]

$$
\begin{gathered}
d s^{2}=\Omega^{2}(r) d U_{+} d V_{+}+r^{2} d \phi_{+}^{2}, \\
\Omega^{2}(r)=\frac{\left(r+r_{+}\right)^{2}}{\kappa_{+}^{2} \Lambda^{2}},
\end{gathered}
$$

and $r$, which is the BTZ radial coordinate, is implicitly defined in terms of $U_{+}$and $V_{+}$in each BTZ patch. In the non-rotating case, on the future horizon $\left(U_{+}=0\right), \partial_{V_{+}}$becomes a Killing vector, and on the past horizon $\left(V_{+}=0\right) \partial_{U_{+}}$becomes a Killing vector. Following Unruh, on the past horizon we require

$$
\mathcal{L}_{\partial_{U_{+}}} \tilde{\phi}=-i \tilde{\omega} \tilde{\phi}
$$

for a wavefunction to have positive energy in the Kruskal sense. Here, $\mathcal{L}_{\partial_{U_{+}}}$is the Lie derivative, $\tilde{\phi}$ is a Kruskal mode, and $\tilde{\omega}>0$. Hence, on the past horizon we have $\tilde{\phi} \sim e^{-i \tilde{\omega} U_{+}}$. This wavefunction is analytic in the lower half $U_{+}$plane, and so we can characterize any positive energy mode by this analyticity. Going back to our modes and wavepacket, this condition tells us to place the branch cut in the upper half $U_{+}$plane. Carrying out a similar analysis on the future event horizon, tells us that the branch cut in $V_{+}$must also be in the UHP. With these branch choices, a BTZ mode with any $\omega$ is a positive energy Kruskal mode. We still have a choice as to the overall Riemann sheet that we are on, i.e. what the phase of 1 is. This choice, along with the choice of kernel, $H(\omega)$, determine whether the wavepacket is concentrated in one asymptotic region versus the other, or in both. For convenience we will choose the phase $1=e^{i 0}$, so that $\ln (1)=0$. (Given a kernel $H(\omega)$ different sheets in which $\ln (1)=2 \pi l$ will result in packets localized in different ways.)

These branch cuts put a restriction on the BTZ coordinate time $t$ as well. From the coordinate transformations (2.11) in region $1_{++}$we have

$$
U_{+}=e^{-\kappa_{+} t} P(r) \quad ; \quad V_{+}=e^{\kappa_{+} t} P(r)
$$

where $P$ is a function of the BTZ radial coordinate. Recall that in Sec. II we showed that one can move between different regions of the BTZ geometry by making discrete translations in the complexified $t$ plane. In particular, as far the as embedding equations (2.5) go translating $t$ by integral multiples of $2 \pi i / \kappa_{+}=i \beta_{*}$ brings us back to the same spacetime point. However, notice that the cuts in $U_{+}$and $V_{+}$arising from the wavepacket, coupled with the relations (4.11), restrict the imaginary parts of $t$. Let $\operatorname{Im}\left(t_{ \pm}\right)$denote the maximum and minimum 
values of the imaginary part of $t$ respectively. Then to stay on a single sheet of the log (namely to avoid the cuts in $U_{+}$and $V_{+}$), we must have

$$
\operatorname{Im}\left(t_{+}\right)-\operatorname{Im}\left(t_{-}\right)<2 \pi / \kappa_{+}=\beta_{*}
$$

Note that the inequality is $\mathrm{a}<$, not $\mathrm{a} \leq$.

- Relation to excursions in the complex plane: We will now work out in detail how these branch choices in the Kruskal $U_{+}$and $V_{+}$planes affect the complexified BTZ coordinates. In the asymptotic region $1_{++}, U_{+}, V_{+}>0$, so no choice of branch is necessary. Near the event horizon the modes making up the wavepacket behave as in (3.12). In $1_{++}$ the outgoing ( $U$ dependent) term can be written

$$
e^{-i \omega U+i \Omega_{H} n U} \sim e^{-\left(i / \kappa_{+}\right) \omega \ln U_{+}+\left(i / \kappa_{+}\right) \Omega_{H} n \ln U_{+}}
$$

where we have left out a constant factor involving $\gamma(4.3)$. Passing through $U_{+}=0$ into $2_{++}$ (where $U_{+}<0$ ) we can explicitly represent the choice of branch by writing the right hand side of the above equation as

$$
e^{-\left(i / \kappa_{+}\right) \omega \ln U_{+}+\left(i / \kappa_{+}\right) \Omega_{H} n \ln U_{+}}=e^{-\frac{\pi \omega}{\kappa_{+}}+\frac{\pi \Omega_{H} n}{\kappa_{+}}} e^{-\left(i / \kappa_{+}\right) \omega \ln \left(-U_{+}\right)+\left(i / \kappa_{+}\right) \Omega_{H} n \ln \left(-U_{+}\right)} .
$$

In BTZ coordinates the factors on the right hand side coming from the branches of the log will get represented via both cuts in the coordinate planes and by an imaginary shift in $t$ (as expected from the excursions in the complex plane that move between BTZ regions).

To characterize this, let us write $t \rightarrow t-i c$ where $c$ is a real constant we wish to find. Rewriting (4.14) in terms of BTZ coordinates we find (note that $0<u<1$ since we are in region $2_{++}$behind the horizon)

$$
e^{-\frac{\pi \omega}{\kappa_{+}}+\frac{\pi \Omega_{H} n}{\kappa_{+}}} e^{-i / \kappa_{+} \omega \ln \left(-U_{+}\right)+i / \kappa_{+} \Omega_{H} n \ln \left(-U_{+}\right)}=(1-u)^{\alpha}(-1)^{\alpha} e^{-i \omega(t-i c)+i \Omega_{H} n(t-i c)},
$$

where we have ignored terms involving the constant $\gamma$ (4.3) which do not affect our analysis. Similarly the ingoing ( $V$ dependent) piece of the mode solution (3.12) leads to

$$
e^{-i / \kappa_{+} \omega \ln \left(V_{+}\right)+i / \kappa_{+} \Omega_{H} n \ln \left(V_{+}\right)}=(1-u)^{-\alpha}(-1)^{-\alpha} e^{-i \omega(t-i c)+\Omega_{H} n(t-i c)},
$$

with no phase shift on the left hand side because $V_{+}>0$ in both $1_{++}$and $2_{++}$. In order to match the Kruskal coordinate phase shifts in BTZ coordinates we must have

$$
(-1)^{\alpha} e^{-\omega c+\Omega_{H} n c}=e^{-\frac{\pi \omega}{\kappa_{+}}+\frac{\pi \Omega_{H} n}{\kappa_{+}}} \quad ; \quad(-1)^{-\alpha} e^{-\omega c+\Omega_{H} n c}=1 .
$$


Solving gives

$$
(-1)^{\alpha}=e^{-\frac{\beta_{*}}{4}\left(\omega-\Omega_{H} n\right)} \quad ; \quad c=\beta_{*} / 4
$$

The first equation amounts to a choice of branch in $r_{*}$, the tortoise coordinate (2.13) constructed from BTZ coordinates. The second equation, amounts to a statement that moving from the asymptotic region $1_{++}$to the region $2_{++}$behind the event horizon is represented in BTZ coordinates as

$$
t \rightarrow t-i \beta_{*} / 4
$$

In other words the ambiguity in shifting $t$ by integral multiples of $i \beta_{*}$ is fixed by the need to match the Kruskal branch cuts. Similarly we find that moving from the right asymptotic region $1_{++}$to the left asymptotic region $1_{+-}$amounts to taking $t \rightarrow t-i \beta_{*} / 2$.

\section{THE CAUCHY HORIZON}

Using the results above we propagate the mode solutions to the inner horizon and show that a wavepacket that is regular at the outer horizon nevertheless has divergent stressenergy on the inner horizon. It is expected that this will lead to large backreaction and an instability in the spacetime. The analysis of this section uses techniques similar to those in section III

To continue our mode solutions from the $1_{++}$region to the $2_{++}$region we must take account of the cuts and shifts in BTZ coordinates that were described in the previous section. Near the event horizon in the $2_{++}$region the mode solutions are given by

$$
\begin{gathered}
\phi_{n}(x)=e^{-i \omega\left(t-i \beta_{*} / 4\right)+i n \phi_{+}+i \Omega_{H} n\left(t-i \beta_{*} / 4\right)}\left[(u-1)^{\alpha} u^{\beta} F\left(\alpha+\beta+h_{+}, \alpha+\beta+h_{-} ; 2 \alpha+1 ; 1-u\right)+\right. \\
\left.\frac{B}{A}(u-1)^{-\alpha} u^{-\beta} F\left(-\alpha-\beta+h_{+},-\alpha-\beta+h_{-} ;-2 \alpha+1 ; 1-u\right)\right]
\end{gathered}
$$

where the factors in front come from the cuts and shifts described in the previous section. In these coordiantes $u=1$ is the event horizon while $u=0$ is the inner horizon.

To study the properties of the solution near the inner horizon we use the linear transformation formulae for hypergeometric functions [15] to write them as functions of $u$. Then the leading terms in $\phi_{n}$ near $u=0$ are

$\phi_{n}^{2_{++}} \approx e^{i n \phi_{-}} e^{-i \omega t+i \Omega_{C} n t} e^{-i \frac{\beta_{*}}{4}\left(i n \Omega_{H}-i \omega\right)}\left\{\left[D(-1)^{\alpha}+E(-1)^{-\alpha}\right] u^{-\beta}+\left[C(-1)^{\alpha}+F(-1)^{-\alpha}\right] u^{\beta}\right\}$, 
(We have switched to the angular coordinate $\phi_{-}(2.19)$ which is appropriate near the inner horizon.) The coefficients, which arise from the transformation formulae and $B / A$ (3.17) are

$$
\begin{aligned}
C & =\frac{\Gamma(2 \alpha+1) \Gamma(-2 \beta)}{\Gamma\left(\alpha-\beta+h_{+}\right) \Gamma\left(\alpha-\beta+h_{-}\right)}, \\
D & =\frac{\Gamma(2 \alpha+1) \Gamma(2 \beta)}{\Gamma\left(\alpha+\beta+h_{+}\right) \Gamma\left(\alpha+\beta+h_{-}\right)}, \\
E & =\frac{\Gamma(2 \alpha) \Gamma\left(-\alpha-\beta+h_{+}\right) \Gamma(-2 \alpha+1) \Gamma(2 \beta)}{\Gamma(-2 \alpha) \Gamma\left(\alpha+\beta+h_{+}\right) \Gamma\left(\alpha-\beta+h_{+}\right) \Gamma\left(-\alpha+\beta+h_{-}\right)}, \\
F & =\frac{\Gamma(2 \alpha) \Gamma\left(-\alpha+\beta+h_{+}\right) \Gamma(-2 \alpha+1) \Gamma(-2 \beta)}{\Gamma(-2 \alpha) \Gamma\left(\alpha+\beta+h_{+}\right) \Gamma\left(\alpha-\beta+h_{+}\right) \Gamma\left(-\alpha-\beta+h_{-}\right)} .
\end{aligned}
$$

Near the inner horizon we can use the set of tortoise coordinates defined in (2.14). In terms of these coordinates, $\tilde{r}_{*} \approx-\frac{1}{2 \kappa_{-}} \ln u$ so $u^{ \pm \beta} \approx \exp \left(\mp i \omega \tilde{r}_{*} \pm i \Omega_{C} n \tilde{r}_{*}\right)$. Using our expression for $(-1)^{\alpha}$ (4.18) we find

$$
\phi_{n}^{2++} \approx e^{i n \phi_{-}}\left[\left(D e^{-\frac{\beta_{*}}{2}\left(\omega-n \Omega_{H}\right)}+E\right) e^{-i \omega \tilde{U}+i \Omega_{C} n \tilde{U}}+\left(C e^{-\frac{\beta_{*}}{2}\left(\omega-n \Omega_{H}\right)}+F\right) e^{-i \omega \tilde{V}+i \Omega_{C} n \tilde{V}}\right],
$$

where $\tilde{U}(\tilde{V})$ dependent terms are waves that move towards the right (left) Cauchy horizon in Fig. 22 Note that even if the wavepacket had been purely ingoing at the outer horizon, scattering from the geometry would have led to both left and right moving pieces at the inner horizon.

Near the inner horizon the wavepacket is

$$
\phi_{w p}(x) \approx e^{i n \phi_{-}}\left[\tilde{f}(\tilde{U}) e^{i \Omega_{C} n \tilde{U}}+\tilde{g}(\tilde{V}) e^{i \Omega_{C} n \tilde{V}}\right],
$$

where the rightmoving piece is

$$
\tilde{f}(\tilde{U})=\int_{-\infty}^{\infty} d \omega\left[D(\omega) e^{-\frac{\beta_{*}}{2}\left(\omega-n \Omega_{H}\right)}+E(\omega)\right] H(\omega) e^{-i \omega \tilde{U}},
$$

and the leftmoving piece is

$$
\tilde{g}(\tilde{V})=\int_{-\infty}^{\infty} d \omega\left[C(\omega) e^{-\frac{\beta_{*}}{2}\left(\omega-n \Omega_{H}\right)}+F(\omega)\right] H(\omega) e^{-i \omega \tilde{V}} .
$$

Using the wavepacket we again find that any divergent contributions to the trace of the stress-energy tensor come from the kinetic term, given by

$$
\begin{gathered}
g^{\mu \nu} \partial_{\mu} \phi_{w p} \partial_{\nu} \phi_{w p}^{*} \approx \frac{2 \Lambda^{2} r_{-}^{2}}{\left(r_{+}^{2}-r_{-}^{2}\right)^{2}} e^{\kappa_{-}(\tilde{V}-\tilde{U})}\left(e^{i \Omega_{C} n(\tilde{U}-\tilde{V})}\left[\tilde{f}^{\prime} \tilde{g}^{\prime *}+i \Omega_{C} n \tilde{f} \tilde{g}^{\prime *}-i \Omega_{C} n \tilde{f}^{\prime} \tilde{g}^{*}+\Omega_{C}^{2} n^{2} \tilde{f} \tilde{g}^{*}\right]+\text { c.c. }\right) \\
-\frac{n^{2}}{r_{+}^{2}-r_{-}^{2}}\left|\phi_{w p}\right|^{2}
\end{gathered}
$$


where the primes are understood in the same way as they were for the event horizon computation. Thus, the overall factor causes a naive divergence in the trace of the stress tensor as $e^{\kappa_{-} \tilde{V}}\left(e^{-\kappa_{-} \tilde{U}}\right)$ as we approach the right (left) Cauchy horizon. As with the event horizon this potential divergence involves an interaction between right and left moving pieces.

Let us examine the behavior of the wavepacket on the left Cauchy horizon. Assume as before that the kernel $H(\omega)$ decays sufficiently quickly at large $\omega$ to use contour integration in (5.9) and (5.10). At the left Cauchy horizon $(\tilde{U} \rightarrow-\infty)$, the potential divergence of the stress tensor can only be suppressed if the rightmoving piece falls off fast enough as $\tilde{U} \rightarrow-\infty$. To study this, we close the contour integral for $\tilde{f}(\tilde{U})$ in the UHP and get contributions from poles in $D(\omega)$ arising from $\Gamma(2 \alpha+1)$ and $\Gamma(2 \beta)$ factors. They occur at

$$
\begin{aligned}
& \omega_{1, k}=i \kappa_{+}(k+1)+\frac{r_{-}}{r_{+} \Lambda} n, \quad k=0,1, \ldots, \\
& \omega_{2, k}=i \kappa_{-} k+\frac{r_{+}}{r_{-} \Lambda} n, \quad k=0,1, \ldots,
\end{aligned}
$$

respectively. Contributions to $\tilde{f}(\tilde{U})$ can also arise from the poles in $E(\omega)$, but those that lie in the UHP are identical to those in $D(\omega)$. In addition, continuity and finiteness of the stress tensor at the event horizon have imposed a condition that any poles in $H(\omega)$ have an imaginary part with magnitude greater than $\kappa_{+}$. Therefore, the dominant behavior for the rightmoving part of the wavepacket, $\tilde{f}(\tilde{U})$ on the left Cauchy horizon $(\tilde{U} \rightarrow-\infty)$ (ignoring oscillating parts), is given by the residues associated with the $k=0$ poles in (5.12). We write this as

$$
\begin{aligned}
\tilde{f}(\tilde{U}) & \left.\sim\left(\operatorname{Res}(E H)+\operatorname{Res}\left(D H e^{-\frac{\beta_{*}}{2}\left(\omega-n \Omega_{H}\right)}\right)\right)\right|_{\omega=\omega_{2,0}} \\
& +\left.\left(\operatorname{Res}(E H)+\operatorname{Res}\left(D H e^{-\frac{\beta_{*}}{2}\left(\omega-n \Omega_{H}\right)}\right)\right) e^{\kappa+\tilde{U}}\right|_{\omega=\omega_{1,0}} .
\end{aligned}
$$

These terms do not decay fast enough on the left Cauchy horizon at $\tilde{U} \rightarrow-\infty$ to suppress the divergence in the trace of the stress tensor coming from the overall factor in (5.11).

Similarly the potential divergence in (5.11) at the right Cauchy horizon $(\tilde{V} \rightarrow \infty)$ can only be suppressed by a sufficiently rapid decay of the leftmoving part $\tilde{g}(\tilde{V})$ of the wavepacket. Closing the contour integral defining (5.10) in the LHP we obtain the leading contributions

$$
\begin{aligned}
\tilde{g}(\tilde{V}) & \sim\left[\operatorname{Res}(F H)+\left.\operatorname{Res}\left(C H e^{-\frac{\beta_{*}}{2}\left(\omega-n \Omega_{H}\right)}\right]\right|_{\omega_{2,0}}\right. \\
& +\left.\exp \left[-2 h_{+} \frac{\left(r_{+}+r_{-}\right)}{\Lambda^{2}} \tilde{V}\right] \operatorname{Res}(F H)\right|_{\omega_{3,0}},
\end{aligned}
$$


where $\omega_{2, k}$ is given above in (5.12) and

$$
\omega_{3, k}=\omega_{R}=-2 i\left(h_{+}+k\right)\left(r_{+}+r_{-}\right) / \Lambda^{2}-n / \Lambda, \quad k=0,1, \ldots
$$

Interestingly the frequencies $\omega_{R}$ are precisely those of half the quasinormal modes. While we

only included the $\omega_{i, 0}$ contributions in the above analysis of the leading terms in the trace of the stress tensor, the higher $k$ frequencies contribute to subleading behaviors. The fact that the quasinormal frequencies contribute only in the leftmoving part of the wavepacket emphasizes again that the perturbation we are constructing is not symmetric between the two asymptotic regions. Thus, the effect we are seeing does not arise from a collision of a symmetric packet, but rather due to the effects of scattering from the geometry and gravitational focusing. As a check on this, one can easily show that even a (non-normalizable) purely ingoing wavepacket at the event horizon leads to divergent behavior on both the left and right Cauchy horizons.

Indeed it is easy to numerically construct a wavepacket with a finite stress tensor on the event horizon, that is exponentially suppressed in the other asymptotic region and nevertheless has a divergent stress tensor on the Cauchy horizon. It is commonly believed that such divergent behavior will cause a gravitational instability at the inner horizon. This is expected to lead to the formation of a singularity and the preservation of cosmic censorship.

\section{ADS/CFT CORRELATORS AND DETECTING THE INSTABILITY}

We have shown how to construct wavepackets with a stress tensor that is finite at the outer horizon, but diverges at the inner horizon. Since the BTZ black hole is asymptotically AdS, there is a description of the spacetime in a dual CFT [20, 21, 22]. In this section we will explore whether and how the CFT is sensitive to the instability at the inner horizon. (Previous work, from a variety of perspectives, on the holographic encoding of physics behind a horizon includes [23, 24, 25, 26, 27, 28].)

In Lorentzian signature the AdS/CFT correspondence is formulated as [29, 30]

$$
\left\langle\phi_{n}\left|T \exp i \int_{\partial} \phi_{0} \mathcal{O}\right| \phi_{n}\right\rangle_{C F T}=Z\left(\phi_{0}\right)
$$

where $Z$ is the string or supergravity partition function with the boundary condition that $\phi \rightarrow \phi_{0}$ on the boundary, $T$ is the time ordering symbol and $\mathcal{O}$ is the operator dual to $\phi$. 
The state $\left|\phi_{n}\right\rangle$ is dual to any normalizable component in the bulk field $\phi(x)$ [29, 30]. We can compute $n$-point functions of $\mathcal{O}$ by taking functional derivatives with respect to $\phi_{0}$ and then setting $\phi_{0}=0$ in the usual way.

As is well known, in Lorentzian signature we have two possible types of modes: normalizable and non-normalizable. Non-normalizable modes are dual to sources in the boundary theory and are also present in the Euclidean formulation of AdS/CFT. A non-normalizable mode can be expressed as

$$
\phi_{n n}(x)=\int d \mathbf{b} K(x, \mathbf{b}) \phi_{0}(\mathbf{b})
$$

where $K(x, \mathbf{b})$ is the bulk-boundary propagator, $x$ are coordinates representing the bulk spacetime and $\mathbf{b}$ lies in the boundary. In the Euclidean formulation of AdS/CFT, these are the only modes present, and by plugging this expression into (6.1) one can build up amplitudes by stringing together bulk-boundary propagators in the appropriate way [21, 22].

Since our instability is a result of a wavepacket we must include normalizable modes and write

$$
\phi(x)=\phi_{w p}(x)+\int d \mathbf{b} K(x, \mathbf{b}) \phi_{\mathbf{0}}(\mathbf{b})
$$

as the expression to be used for computing correlators. Such a classical wavepacket should be related to a suitable coherent state in the boundary theory. Inserting this expression into (6.1), taking one functional derivative and setting the source to zero gives a contribution to the CFT 1-point function which goes as

$$
\left\langle\phi_{w p}|O(\mathbf{b})| \phi_{w p}\right\rangle \sim \int d^{3} x \sqrt{-g} g^{\mu \nu} \partial_{\mu} \phi_{w p}(x) \partial_{\nu} K(x, \mathbf{b})
$$

In view of the extended Penrose diagram Fig. [1 with multiple asymptotic regions and horizons, the key question is what regions are included in the integration over the bulk and which boundary components contribute.

The obvious application of the correspondence as stated in [21, 22] would suggest that we should integrate in the bulk over the entire spacetime, including the infinitely repeating regions in Fig. 1 (for relevant discussions see [5]). The spacetime contains an infinite number of disconnected boundaries, so the dual CFT would presumably contain infinitely many disconnected components. However, boundary conditions would also have to be specified at the naked timelike singularities beyond each inner horizon. This is not very palatable. In any case, the instability that we have found at the inner horizon is expected to close off the 
spacetime there and thus limit the range of integration to include only the regions shown in Fig. 2,

In [6, 7] prescriptions for computing the vacuum correlation functions were given by appealing to analytic continuation from the Euclidean section. One prescription involves integrating the bulk point, $x$, over all of regions $1_{+ \pm}$and $2_{+ \pm}$shown in Fig. 2, up to, but not including the full Cauchy horizon. This can be simply expressed by using the Poincare disc coordinates or the outer Kruskal coordinates $\left(U_{+}, V_{+}\right)$that were described earlier, since these coordinates cover same range, namely all of Fig. 2 except precisely the inner horizon.

In an alternative prescription, derived by a different analytic continuation of the same Euclidean section, we integrate over only the regions outside the event horizon $\left(1_{+ \pm}\right)$but over a contour in the complex $t$ plane which we shall describe below. In effect we use BTZ coordinates in the outer region and exploit the excursions in the complex plane described earlier to move from $1_{++}$to $1_{+-}$. It was proposed in [6, 7] that the parts of the contour that move purely in the imaginary time direction should be thought of as encoding the physics behind the horizon.

In the case of the vacuum correlation functions, both of these prescriptions are different analytic continuations of the same Euclidean integral, and therefore they give the same result. It was therefore claimed in [6, 7] that the integral over only the external regions (plus the imaginary time parts of the integration contour) encode information from behind the horizon. However, in the vacuum situation studied in these papers nothing special happens behind the horizon, so a skeptic might have read the result in the opposite direction: the AdS/CFT prescription that integrates behind a horizon is really only sensitive to information that is accessible to the asymptotic observer. Our ability to include a wavepacket that is regular at the outer horizon and breaks down at the inner horizon provides an interesting opportunity to test how a dramatic phenomenon localized behind the horizon is detected by the AdS/CFT correspondence. Of course, when a state is present, we cannot perform a continuation to the Euclidean section; so it is not possible to derive a prescription for computing amplitudes via such analytic continuations. Instead we will adopt the prescriptions derived by [6, 7] in the vacuum since they should not change much if a small perturbation is introduced in the spacetime. Of course, our perturbation has dramatic effects at the inner horizon and we are precisely interested in the resulting breakdown of the semiclassical approximation of the AdS/CFT correspondence. 


\section{A. General considerations}

We are interested in seeing some sign of the breakdown of the semiclassical AdS/CFT correspondence due to the inner horizon instability. The first step is to demonstrate that the contribution to CFT correlators from the vicinity of the outer horizon is finite and well defined. Then we will study the contribution from the inner horizon. We will be principally interested in the 1-point function given by the integral (6.4). Potential breakdowns in this integral can arise from the measure factor $\sqrt{-g}$ and from the coordinate invariant piece $g^{\mu \nu} \partial_{\mu} \phi_{w p} \partial_{\nu} K$. Here we will study the behavior of the integrand at the outer and inner horizons and in the next section we will study the full integral.

(i) The outer horizon: Let us examine the contribution to the 1-point function in (6.4) from the vicinity of the outer horizon in the region $1_{++}$. (The qualitative behavior will be similar in $\left.1_{+-}.\right)$The expression for the bulk-boundary propagator can be obtained from the $A d S_{3}$ bulk-boundary propagator by a sum over images. When both the boundary point and the bulk point are contained in region $1_{++}$it is given by [5, 6, 7, 14$]$

$$
K^{1_{++} 1_{++}}\left(x, b^{\prime}\right)=c \sum_{l=-\infty}^{\infty}\left\{\sqrt{u} \cosh \left(\frac{r_{-}}{\Lambda^{2}} \Delta t-\frac{r_{+}}{\Lambda} \Delta \bar{\phi}_{l}\right)-\sqrt{u-1} \cosh \left(\frac{r_{+}}{\Lambda^{2}} \Delta t-\frac{r_{-}}{\Lambda} \Delta \bar{\phi}_{l}\right)\right\}^{-2 h_{+}},
$$

where $c$ is a constant, $\Delta t=t-t^{\prime}$ and $\Delta \bar{\phi}_{l}=\bar{\phi}-\bar{\phi}^{\prime}+2 \pi l$. The spacetime integral giving the CFT 1-point function (6.4) involves a measure factor $\sqrt{-g}$ and a coordinate invariant expression of the form $g^{\mu \nu} \partial_{\mu} \phi_{w p} \partial_{\nu} K$.

This invariant piece can be evaluated near the event horizon, giving (ignoring for now the sum over $l$ in the propagator (6.5) and switching to the $\phi_{+}=\bar{\phi}-\Omega_{H} t$ coordinate)

$$
\begin{aligned}
g^{\mu \nu} \partial_{\mu} \phi_{w p}(x) \partial_{\nu} K\left(x, b^{\prime}\right) \approx- & 2 h_{+}\{\ldots\}^{-2 h_{+}-1} e^{i n \phi_{+}} \frac{r_{+}}{r_{+}^{2}-r_{-}^{2}} \times \\
\left(e ^ { - \kappa _ { + } r _ { * } } \left\{f^{\prime} e^{i \Omega_{H} n U}[\right.\right. & \left.\left.\exp \left(\kappa_{+} \Delta t-\frac{r_{-} \Delta \phi_{+}}{\Lambda}\right)\right]-g^{\prime} e^{i \Omega_{H} n V} \exp \left[-\left(\kappa_{+} \Delta t-\frac{r_{-} \Delta \phi_{+}}{\Lambda}\right)\right]\right\} \\
+e^{-\kappa_{+} r_{*}} i \Omega_{H} n\left\{f e^{i \Omega_{H} n U}[\right. & \left.\left.\exp \left(\kappa_{+} \Delta t-\frac{r_{-} \Delta \phi_{+}}{\Lambda}\right)\right]-g e^{i \Omega_{H} n V} \exp \left[-\left(\kappa_{+} \Delta t-\frac{r_{-} \Delta \phi_{+}}{\Lambda}\right)\right]\right\} \\
+ & {\left[e^{i \Omega_{H} n U}\left(-f^{\prime}-i \Omega_{H} n f\right)+e^{i \Omega_{H} n V}\left(g^{\prime}+i \Omega_{H} n g\right)\right] \cosh \left(\frac{r_{+} \Delta \phi_{+}}{\Lambda}\right) } \\
& \left.+\sinh \left(\frac{r_{+} \Delta \phi_{+}}{\Lambda}\right) \frac{i n}{\Lambda}\left(f e^{i \Omega_{H} n U}+g e^{i \Omega_{H} n V}\right)\right),
\end{aligned}
$$


where

$$
\{\ldots\}=\sqrt{u} \cosh \left(\frac{r_{+}}{\Lambda} \Delta \phi_{+}\right)-\sqrt{u-1} \cosh \left(\kappa_{+} \Delta t-\frac{r_{-}}{\Lambda} \Delta \phi_{+}\right) .
$$

Each $\Delta \phi_{+}$in these expressions should really be a $\Delta \phi_{+, l}$ with the $2 \pi l$ shifts coming from the propagator. We are neglecting the sum over $l$ because this does not contribute to any divergent behavior at the outer horizon.

It is easiest to examine the behavior of the integral (6.4) near the event horizon in the outer Kruskal coordinates $\left(U_{+}, V_{+}, \phi_{+}\right)$defined in (2.15), as the coordinate system is well defined on the event horizon and it is easier to perform the integrals. The metric becomes

$$
\begin{aligned}
d s^{2} & =\Omega^{2}(r) d U_{+} d V_{+}+r^{2}\left(N^{\phi_{+}} d t+d \phi_{+}\right)^{2}, \\
\Omega^{2}(r) & =\frac{\left(r^{2}-r_{-}^{2}\right)\left(r+r_{+}\right)^{2}}{\kappa_{+}^{2} r^{2} \Lambda^{2}}\left(\frac{r-r_{-}}{r+r_{-}}\right)^{r_{-} / r_{+}}, \\
N^{\phi_{+}} & =\frac{r_{-}}{\Lambda r_{+} r^{2}}\left(r^{2}-r_{+}^{2}\right),
\end{aligned}
$$

where it is understood that $t$ and $r$ are now implicit functions of $U_{+}$and $V_{+}$. In these coordinates $\sqrt{-g}$ is finite along the whole event horizon. In addition, one can check that $\{\ldots\}$ (6.7) is finite along both the past and future event horizons. Therefore, any possible divergent behavior must come from the terms in (6.6).

Examining (6.6) and using (4.2) we see that the only possibly divergent terms have the general form of

$$
f\left(\text { or } f^{\prime}\right) e^{i \Omega_{H} n U} e^{\kappa_{+} \Delta t-\frac{r_{-} \Delta \phi_{+}}{\Lambda}} e^{-\kappa_{+} r_{*}} \sim \frac{f(U)}{U_{+}},
$$

or

$$
g\left(\text { or } g^{\prime}\right) e^{i \Omega_{H} n V} e^{-\kappa_{+} \Delta t+\frac{r_{-} \Delta \phi_{+}}{\Lambda}} e^{-\kappa_{+} r_{*}} \sim \frac{g(V)}{V_{+}} .
$$

(We can treat the derivative terms the same way, because we are only interested in the exponential part of $f$ and $g$.) On the past event horizon the outgoing piece (6.11) is finite. To check the ingoing piece (6.12), recall that $g(V) \sim e^{\kappa+V}$ on the past event horizon. We find

$$
\frac{g(V)}{V_{+}} \sim \frac{e^{\kappa_{+} V}}{V_{+}} \sim 1,
$$

and so is finite. A similar computation shows both terms to be finite on the future event horizon as well. Thus, the integrand is finite along the entire event horizon and the integral for the 1-point function does not receive any divergent contributions from the event horizon. 
(ii) The inner horizon: We can carry out a similar set of calculations near the Cauchy horizon. When the bulk point is the region $2_{++}$we can find the bulk-boundary propagator by an analytic continuation [5, 6, 7]

$$
K^{2_{++} 1_{++}}\left(x, b^{\prime}\right)=c^{\prime} \sum_{l=-\infty}^{l=\infty}\left\{\sqrt{u} \cosh \left(\frac{r_{-}}{\Lambda^{2}} \Delta t-\frac{r_{+}}{\Lambda} \Delta \bar{\phi}_{l}\right)-\sqrt{1-u} \sinh \left(\frac{r_{+}}{\Lambda^{2}} \Delta t-\frac{r_{-}}{\Lambda} \Delta \bar{\phi}_{l}\right)\right\}^{-2 h_{+}} .
$$

The coordinate invariant part of the integrand in (6.4), i.e., $g^{\mu \nu} \partial_{\mu} \phi_{w p} \partial_{\nu} K$, evaluated near the Cauchy horizon is (again ignoring the sum in the propagator for now and switching to $\phi_{-}$defined in (2.19) )

$$
\begin{array}{r}
g^{\mu \nu} \partial_{\mu} \phi_{w p}(x) \partial_{\nu} K\left(x, b^{\prime}\right) \approx-2 h_{+}\{\ldots\}^{-2 h_{+}-1} e^{i n \phi_{-}} \frac{r_{-}}{r_{+}^{2}-r_{-}^{2}} \times \\
\left(e^{\kappa-\tilde{r}_{*}}\left[-\tilde{f}^{\prime} e^{i \Omega_{C} n \tilde{U}} \exp \left(-\kappa_{-} \Delta t-\frac{r_{+}}{\Lambda} \Delta \phi_{-}\right)+\tilde{g}^{\prime} e^{i \Omega_{C} n \tilde{V}} \exp \left(\kappa_{-} \Delta t+\frac{r_{+}}{\Lambda} \Delta \phi_{-}\right)\right]\right. \\
+e^{\kappa \tilde{r}_{*}} i \Omega_{C} n\left[-\tilde{f} e^{i \Omega_{C} n \tilde{U}} \exp \left(-\kappa_{-} \Delta t-\frac{r_{+}}{\Lambda} \Delta \phi_{-}\right)+\tilde{g} e^{i \Omega_{C} n \tilde{V}} \exp \left(\kappa_{-} \Delta t+\frac{r_{+}}{\Lambda} \Delta \phi_{-}\right)\right] \\
-\left[e^{i \Omega_{C} n \tilde{U}}\left(-\tilde{f}^{\prime}-i \Omega_{C} n \tilde{f}\right)+e^{i \Omega_{C} n \tilde{V}}\left(\tilde{g}^{\prime}+i \Omega_{C} n \tilde{g}\right) \sinh \left(\frac{r_{-}}{\Lambda} \Delta \phi_{-}\right)\right. \\
\left.-\frac{i n}{\Lambda}\left(\tilde{f} e^{i \Omega_{C} n \tilde{U}}+\tilde{g} e^{i \Omega_{C} n \tilde{V}}\right) \cosh \left(\frac{r_{-}}{\Lambda} \Delta \phi_{-}\right)\right),
\end{array}
$$

with

$$
\{\ldots\}=\sqrt{u} \cosh \left(\kappa_{-} \Delta t+\frac{r_{+}}{\Lambda} \Delta \phi_{-}\right)+\sqrt{1-u} \sinh \left(\frac{r_{-}}{\Lambda} \Delta \phi_{-}\right) .
$$

It is easiest to examine the behavior of the 1-point function integral (6.4) near the inner horizon using the inner Kruskal coordinates $\left(U_{-}, V_{-}, \phi_{-}\right)$given in (2.19] 2.21). The metric is given by

$$
\begin{gathered}
d s^{2}=\tilde{\Omega}^{2}(r) d U_{-} d V_{-}+r^{2}\left(N^{\phi_{-}} d t+d \phi_{-}\right)^{2}, \\
\tilde{\Omega}^{2}(r)=\frac{\left(r_{+}^{2}-r^{2}\right)\left(r+r_{-}\right)^{2}}{\kappa_{-}^{2} r^{2} \Lambda^{2}}\left(\frac{r_{+}-r}{r_{+}+r}\right)^{r_{+} / r_{-}}, \\
N^{\phi_{-}}=\frac{r_{+}}{\Lambda r_{-} r^{2}}\left(r^{2}-r_{-}^{2}\right),
\end{gathered}
$$

and the determinant of the metric and the overall factor (6.16) $\{\ldots\}$ are finite on the inner horizon. Near the Cauchy horizon we have

$$
\begin{gathered}
r-r_{-} \approx-2 r_{-}\left(\frac{r_{+}-r_{-}}{r_{+}+r_{-}}\right)^{r_{+} / r_{-}} U_{-} V_{-}, \\
e^{\kappa-t}=\sqrt{\frac{-U_{-}}{V_{-}}}
\end{gathered}
$$


(note that $U_{-} / V_{-}<0$ in region $2_{++}$).

In a similar analysis to the one performed near the event horizon, we find the leading (potentially divergent) terms near the Cauchy horizon have the form

$$
\tilde{f}\left(\text { or } \tilde{f}^{\prime}\right) e^{i \Omega_{C} n \tilde{U}} e^{\kappa_{-} \tilde{r}_{*}} e^{-\kappa_{-} \Delta t-\frac{r_{+}}{\Lambda} \Delta \phi_{-}} \sim \frac{\tilde{f}(\tilde{U})}{U_{-}} e^{\kappa_{-} t^{\prime}-\frac{r_{+}}{\Lambda} \Delta \phi_{-}},
$$

or

$$
\tilde{g}\left(\text { or } \tilde{g}^{\prime}\right) e^{i \Omega_{C} n \tilde{V}} e^{\kappa_{-} \tilde{r}_{*}} e^{\kappa_{-} \Delta t+\frac{r_{+}}{\Lambda} \Delta \phi_{-}} \sim \frac{\tilde{g}(\tilde{V})}{V_{-}} e^{-\kappa_{-} t^{\prime}+\frac{r_{+}}{\Lambda} \Delta \phi_{-}} .
$$

On the left Cauchy horizon $\left(U_{-}=0\right)$ the leftmoving piece (6.23) is finite since we know that $\tilde{g}(\tilde{V})$ itself is well behaved and $V_{-}$is nonzero there. Using (15.13), the rightmoving piece (6.22) goes as

$$
\begin{aligned}
\frac{\tilde{f}(\tilde{U})}{U_{-}} & \sim \frac{\left.\left(\operatorname{Res}(E H)+\operatorname{Res}\left(D H e^{-\frac{\beta_{*}}{2}\left(\omega-n \Omega_{H}\right)}\right)\right)\right|_{\omega=\omega_{2,0}}}{U_{-}} \\
& +\frac{\left.\left(\operatorname{Res}(E H)+\operatorname{Res}\left(D H e^{-\frac{\beta_{*}}{2}\left(\omega-n \Omega_{H}\right)}\right)\right) e^{\kappa_{+} \tilde{U}}\right|_{\omega=\omega_{1,0}} \sim U_{-}^{-1}+U_{-}^{\kappa_{+} / \kappa_{-}-1}}{U_{-}} .
\end{aligned}
$$

This diverges as $U_{-} \rightarrow 0$ at the horizon. Similarly, on the right Cauchy horizon $\left(V_{-}=0\right)$ the rightmoving piece (6.22) is finite, while the leftmoving piece (6.23) diverges as

$$
\begin{aligned}
\frac{\tilde{g}(\tilde{V})}{V_{-}} & \sim \frac{\left[\operatorname{Res}(F H)+\left.\operatorname{Res}\left(C H e^{-\frac{\beta_{*}}{2}\left(\omega-n \Omega_{H}\right)}\right]\right|_{\omega_{2,0}}\right.}{V_{-}} \\
& +\frac{\left.\exp \left[-2 h_{+} \frac{\left(r_{+}+r_{-}\right)}{\Lambda^{2}} \tilde{V}\right] \operatorname{Res}(F H)\right|_{\omega_{3,0}}}{V_{-}} \sim V_{-}^{-1}+V_{-}^{\frac{2 h_{+} r_{-}}{r_{+}-r_{-}}-1}
\end{aligned}
$$

To summarize, we have seen that the leading behavior of the integrand in the AdS/CFT expression for the CFT 1-point function (6.4) is finite on the event horizon and divergent on the inner horizon. To see whether this leads to a breakdown or divergence in the CFT 1-point function we have to examine the full integral in (6.4) and understand how it behaves in the different prescriptions for integrating over the the BTZ spacetime that were discussed above and in [6, 7].

\section{B. Computing the 1-point function}

As discussed before there are different prescriptions for carrying out the 1-point function integral in (6.4). The obvious procedure would have been to integrate over the entire spacetime, or at least over all of the regions in Fig. 2 including the Cauchy horizon. As 
we discussed, becaused of the timelike singularities beyond the Cauchy horizon, this is potentially problematic. The second procedure, from [7], is derived by analytic continuation in the vacuum case and requires an integral up to, but not including the Cauchy horizon. This can be done either in the disc coordinates or in the outer Kruskal coordinates that were described before. Finally, a third prescription, also derived in [7] in the vacuum case via analytic continuation, integrates up to and including the outer horizon in both asymptotic regions and over part of the complexified BTZ coordinate plane. Our goal will be to show how all of these semiclassical procedures break down because of the presence of the wavepacket in our setup and the resulting instability of the inner horizon.

(i) Integrating through the Cauchy horizon: Suppose that the correct region of integration includes the Cauchy horizon in $2_{++}$and in $2_{+-}$. This could either mean that we integrate over the entire spacetime (as one would naively suppose) or that we integrate over all of the regions in Fig. 2 including the Cauchy horizon. Near the left Cauchy horizon, the leading behavior of the integral is

$$
\int d^{3} x \sqrt{-g} g^{\mu \nu} \partial_{\mu} \phi_{w p}(x) \partial_{\nu} K(x, \mathbf{b}) \sim \int d V_{-} \int d \phi_{-} \int^{0} d U_{-}\{\ldots\}^{-2 h_{+}-1} U_{-}^{-1},
$$

where the lower limit is unimportant for our considerations. Using our expression for $\{\ldots\}$ (6.16) and the transformation equations (6.20, 6.21), we find

$$
\begin{aligned}
& \int d V_{-} \int d \phi_{-} \int^{0} d U_{-}\{\ldots\}^{-2 h_{+}-1} U_{-}^{-1} \\
\sim & \int d V_{-} \int d \phi_{-} \int^{0} d U_{-} \frac{1}{U_{-}\left[U_{-} e^{-\kappa_{-} t^{\prime}-\frac{r_{+}}{\Lambda} \Delta \phi_{-}}+V_{-} e^{\kappa-t^{\prime}+\frac{r_{+}}{\Lambda} \Delta \phi_{-}}+\sinh \left(\frac{r_{-}}{\Lambda} \Delta \phi_{-}\right)\right]^{2 h_{+}+1}} \\
\sim & \left.\int d V_{-} \int d \phi_{-} \ln \left(-U_{-}\right)\right|^{0} \sim \infty,
\end{aligned}
$$

where we have only kept the most divergent piece. A similar calculation shows that the integral diverges logarithmically in $V_{-}$near the right Cauchy horizon as well.

In 6] a potential divergence was found coming from the region near the non-rotating BTZ singularity. This arose because of the sum on images in the bulk-boundary propagator. The situation here is very different. It was already shown in [7] that the sum on images does not lead to divergences in correlation functions from regions near the Cauchy horizon. The same applies here. We are uncovering a different phenomenon associated with the focusing of the wavepacket at the inner horizon. 
In 6] the potential divergence arising from the sum on images could be regulated by an $i \epsilon$ prescription derived from Euclidean continuation of the bulk-boundary propagator. In addition there were cancellations between some contributions to the correlation function integrals at the past and future BTZ singularities. Such regularizations and cancellations cannot occur here. First, let us consider whether divergences from the right and left Cauchy horizons could cancel, and second, whether divergences from the Cauchy horizon in $2_{++}$ could cancel those in $2_{+-}$. From $(6.22,6.23)$ we see that the two pieces have different dependences on the boundary (primed) coordinates. Therefore, for general values of $t^{\prime}, \phi_{-}^{\prime}$ the contributions from the right and left Cauchy horizons are different and the two terms cannot cancel. Turning to the other possibility it is easy to show that the wavepacket is not generically symemtric between the $2_{++}$and $2_{+-}$regions and therefore there are no cancellations.

Since the divergences will not cancel we can attempt to regulate them. One might try to use a Cauchy Principal Value prescription for integrating through the inner horizon. However, this regularization prescription will fail for higher derivative terms that we have not explicitly included in the present analysis. The other alternative is to choose an $i \epsilon$ prescription to define the 1-point function integrals (6.4) since a similar prescription rendered integrals in the non-rotating vacuum case finite [6]. However, because of the absence of a Euclidean continuation in our case, and the fact that our wavepackets are complex as well as containing both left and rightmoving ( $U_{-}$and $V_{-}$dependent) pieces, there is no obvious principled approach to regulating our integrals in this way without introducing pinched singularities.

In addition, stringy $\left(\alpha^{\prime}\right)$ corrections to the supergravity action will contain terms with more and higher derivatives. These terms will lead to even worse divergences in the integral. The essential lesson here is that backreaction and strong curvature effects will be important near the inner horizon and we should expect to need the full string theory, not just the semiclassical supergravity approximation. Since the dual CFT is finite and unitary, the properly computed 1-point function must be finite. Thus, we interpret the divergence of the integral (6.4) as a breakdown in the semiclassical approximation to the AdS/CFT correspondence. In this prescription it is clear that correlation functions are sensitive to the physics near the inner horizon. 
(ii) The prescription in disc coordinates: In [7] a prescription for computing AdS/CFT correlators was found (via analytic continuation from the Euclidean section) that integrates over the regions shown in Fig. 2. This is conveniently expressed in Poincare disc coordinates (see 7] for the details of this coordinate system) which cover the same regions as the outer Kruskal coordinates $\left(U_{+}, V_{+}, \phi_{+}\right)$. These coordinates cover all of $1_{+ \pm}$and all of $2_{+ \pm}$except for the Cauchy horizon. In other words, the range for the BTZ radial coordinate is $r_{-}<r<\infty$. (This differs from the the analysis above in that the actual inner horizon is excluded. Since this is a set of measure zero it can only matter if the integrand in (6.4) diverges there.)

Above, we saw that the integral contributing to the 1-point function picked up a divergence from the contribution on the Cauchy horizon. Here we must omit the actual horizon, but include points that are infinitesimally separated from it. ¿From (6.27) the resulting behavior near the left Cauchy horizon is

$$
\left.\int d V_{-} \int d \phi_{-} \int^{-\epsilon} d U_{-}\{\ldots\}^{-2 h_{+}-1} U_{-}^{-1} \sim \ln \left(-U_{-}\right)\right|^{-\epsilon} \sim \ln \epsilon
$$

where we cut off the Cauchy horizon by limiting the integral to the range $-U_{-} \geq \epsilon$. This integral is again ill-defined as $\epsilon \rightarrow 0$. In addition, stringy effects and higher derivative interactions will produce terms that go like $1 / \epsilon, 1 / \epsilon^{2}, \ldots$ which will not be suppressed relative to the semiclassical supergravity approximation. As above, this integral is sensitive to the instability at the inner horizon and the semiclassical approximation to the AdS/CFT correspondence breaks down. Also as discussed above, these divergences do not cancel between different horizons and cannot be regulated away in a straightforward manner.

(iii) The prescription in BTZ coordinates: In the other prescription given in 6, 7] we are instructed to integrate using BTZ coordinates only over the region outside the event horizon, but over a contour in the complex $t$ plane. Since we are only integrating over the exterior region, the way in which the correlator can see the instability inside the horizon is more subtle.

In this prescription (6.4) becomes

$$
\left\langle\phi_{w p}|O(\mathbf{b})| \phi_{w p}\right\rangle \sim \int_{C} d^{3} x \sqrt{|g|} g^{\mu \nu} \partial_{\mu} K_{C}(x, \mathbf{b}) \partial_{\nu} \phi_{w p, C}(x)
$$

where the $t$ integral is carried out over the contour $C$ shown in Fig. 3, and we integrate only over the asymptotic regions $1_{+ \pm}$down to the event horizon. Both $\phi_{w p}$ and $K$ are defined 
over the complex values of $t$. For the bulk-boundary propagator, this analytic continuation is straightforward and takes us over the different possible choices of bulk and boundary points, as well as the Euclidean bulk-boundary propagator. However, a problem arises when we consider the presence of $\phi_{w p}$ in the analytically continued integral. Recall, due to the presence of the branch cuts in Kruskal coordinates, we found a restriction on the imaginary values of BTZ time, $t$, given by (4.12). However, the integration over the vertical sections of the contour shown in Fig. 3 violates this restriction and hence crosses the branch cut in general. As a result the integral is not well defined as is since we will be integrating over the same spacetime point with different values for the wavepacket $\phi_{w p}$. By shifting the cuts the best we can do is to have the both the upper horizontal section and the lower end point of the contour just touch the cuts. While we could try to define the integral anyway via a suitable infinitesimal deformation of the contour, it is not clear exactly what deformation to use and what its physical justification would be.

Notice that it is precisely the presence of the vertical sections of the contour, representing the entanglement between the dual CFTs living on the two BTZ boundaries, which lead to the breakdown of the integral. The horizontal sections can be well defined within the restrictions due to the branch cuts. Since we understand the breakdown as arising from an instability at the inner horizon it is tempting to conclude that the presence of an entanglement between the two boundary CFTs can encode information from behind the horizon of the black hole.

\section{Remarks}

We have seen that the inner horizon instability is picked up when we are integrating over the region including the Cauchy horizon as a divergence arising ultimately from the focusing effect. When integrating only over the outer region, the required contour leads to an integral that is not defined. Of course, if we were to do the 1-point function computation in the boundary theory itself, we must get a finite, well-defined answer. Thus, we interpret the problems with the bulk prescription as a breakdown of the semiclassical approximation to the AdS/CFT correspondence and as a signal that backreaction must be accounted for. It would be natural, for example, for the integration contour in Fig. 3 to be modified by

backreaction. For example, since the temperature of the black hole determines the lower 


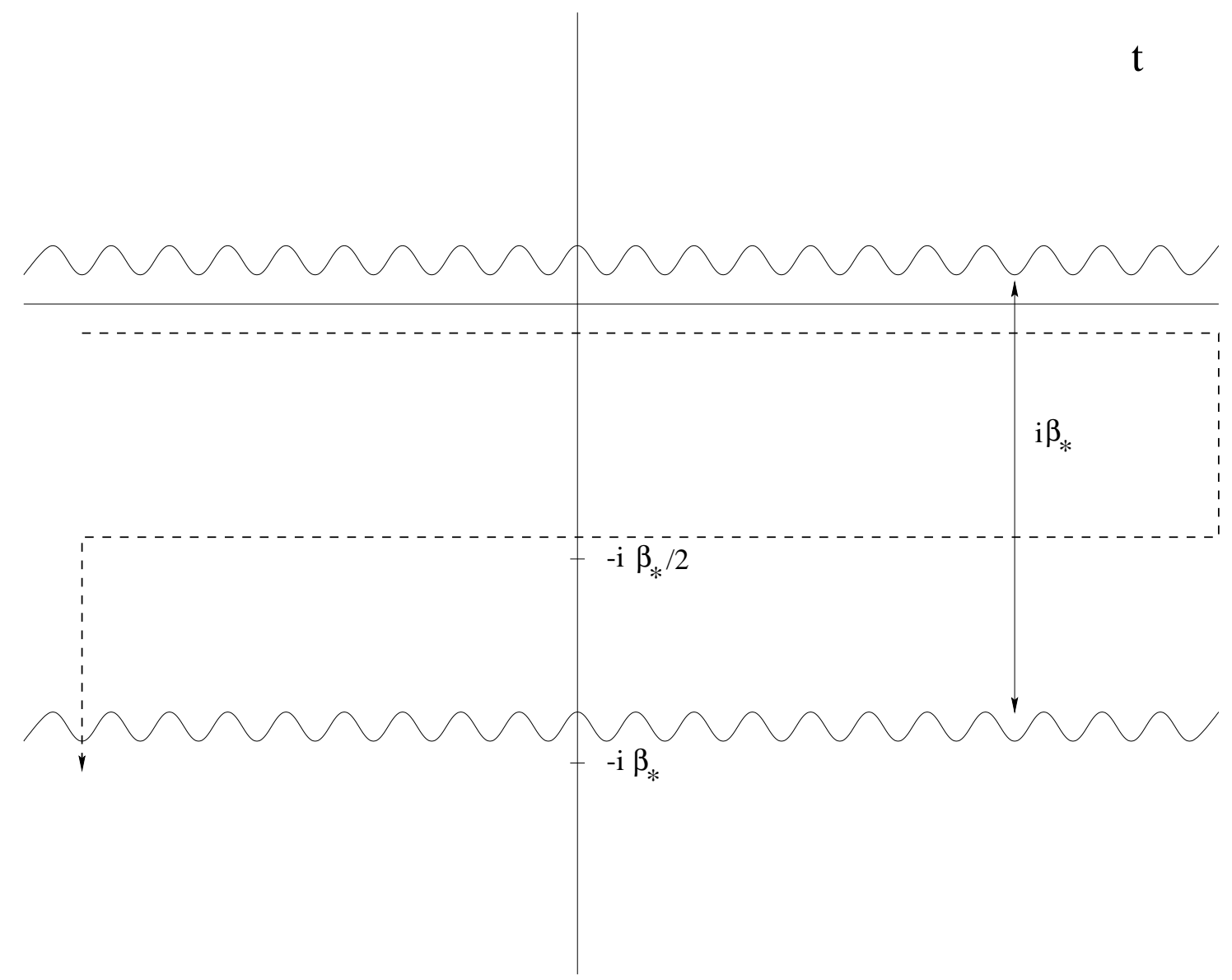

FIG. 3: The complex $t$ contour for integration in BTZ coordinates. The wavy lines are the branch cuts that restrict the range of imaginary time. While choosing different locations for the cuts can move these lines, the contour will always either cross or hit the cuts.

endpoint of the contour in the complex time plane, in the context where we have thrown in an additional wavepacket one might ask whether the correct contour involves the temperature seen by an asymptotic observer before or after the wavepacket has entered the black hole. More generally, the dynamical process of equilibration should be involved. In this regard, previous studies of backreaction and its effects on black hole radiance (see, e.g., [31]) may be interesting.

We have mostly focused on the physics of the inner horizon of rotating black holes. In fact, a similar set of calculations can be carried out for the non-rotating BTZ black hole and establishes that a normalizable wavepacket has large backreaction at the singularity. The analysis in BTZ coordinates is similar to the above and we find that the contour crosses the 
cuts. For the prescription in Kruskal coordinates a general derivative interaction will possess two types of divergences, those coming from the sum over images in the bulk-boundary propagator at the singularity and those coming from the blueshifting of the wavepacket. In 6] a regularization of the divergences coming from the bulk-boundary propagator was found through analytic continuation from the Euclidean section that rendered integrals finite. This regularization amounts to adding a small, imaginary piece, $i \epsilon$ to the radial coordinate $r$ and then taking the limit $\epsilon \rightarrow 0$ at the end of the calculation. Since a normalizable wavepacket is not regular in the Euclidean section, it is difficult to unambiguously define a regularization for its contribution to CFT correlators. Without regularization we find that higher derivative interactions diverge and stringy corrections are no longer suppressed. If we try to use the regularization from [6], we find that since the wavepacket is in general complex, any terms that contain the wavepacket and its complex conjugate will have opposite $i \epsilon$ prescriptions and pinched singularities seem to occur. It is important to emphasize that the rotating case is fundamentally different. Without the wavepacket, there are no divergences associated with the inner horizon and so no regularization is necessary []], in contrast to the singularity in the non-rotating case. Therefore, in the rotating case, there is no suggestion at all from the Euclidean section as to how to regulate the divergences that are present.

One should interpret AdS/CFT calculations in the BTZ background as computing CFT expectation values in a state that entangles the two otherwise disconnected boundary CFT components [6, 27, 30, 32]

$$
|\Psi\rangle=\frac{1}{\sqrt{Z}} \sum_{n} e^{-\frac{\beta_{*} E_{n}}{2}-\frac{\mu \beta_{*} l_{n}}{2}}\left|E_{n}, l_{n}\right\rangle_{1} \otimes\left|E_{n}, l_{n}\right\rangle_{2},
$$

where $Z$ is a normalization factor, $\left|E_{n}, l_{n}\right\rangle$ is an energy and angular momentum eigenstate in one of the CFTs and $\mu$ is the chemical potential related to the angular velocity of the outer horizon of the black hole. The entanglement is between the CFTs living on the two disconnected boundaries of the black hole (i.e. in $1_{++}$and $1_{+-}$). If we only insert operators in one CFT, we can trace over states in the second CFT, and obtain a thermal expectation value for an $n$-point function $G_{n}$

$$
G_{n}=\operatorname{Tr}\left\{e^{-\beta_{*} H} T\left[\mathcal{O}\left(t_{1}\right), \ldots \mathcal{O}\left(t_{n}\right)\right]\right\},
$$

where we have suppressed the angular dependence. Writing out this trace in terms of evolution operators one can obtain the usual thermal contour in [6]. In our case, we have 
perturbed away from thermal equilibrium and our state is no longer given by (6.30). Tracing over the states in the second CFT leads to an expression of the form

$$
G_{n}=\operatorname{Tr}\left\{\rho T\left[\mathcal{O}\left(t_{1}\right), \ldots \mathcal{O}\left(t_{n}\right)\right]\right\}
$$

where $\rho$ is some non-equilibrium density matrix. Notably it will include initial correlations. It is no longer possible in general to obtain the usual thermal contour [33], which is precisely what we expected. In principle, one could carry out this computation in the boundary theory and so hope to obtain information about the resolution of the inner horizon instability (and the resulting singularity) using holography.

\section{CONCLUSIONS AND DISCUSSION}

We showed that the addition of normalizable modes in the rotating BTZ black hole opens up a wealth of new physics. At the classical level, we found it necessary to construct wavepackets to regularize behavior on the outer horizon. Even with this regularization a focusing instability is always present at the inner horizon and so it is likely that a singularity forms there. Continuity, single-valuedness and positive energy of wavepackets as we move between black hole regions control the location of branch cuts in the complexified coordinate planes. We showed that AdS/CFT correlators are sensitive to the inner horizon instability and that the prescription that integrates only outside the black hole learns about the black hole interior through these cuts. This result reinforces observations in [6, 7, 8, 9, 10] that point to the importance of the complexified coordinate plane as a repository of physical information in time-dependent backgrounds.

In this regard, it is interesting that the boundary of the Euclidean black hole is a single, connected torus, and that one can also view motion in the imaginary time direction as connecting the two asymptotic regions and boundaries in the Lorentzian section. Thus, the vertical parts of the contour in Fig. 3 correspond to moving along this torus from one boundary CFT to the other. It is worth noting that the analytic continuation in [6, 7] that led to this contour prescription is not in fact unique. For example, for the purpose of computing CFT correlation functions in the Hartle-Hawking vacuum for the BTZ black hole, one could have used a contour in Fig. 3 in which the lower horizontal section was at $\operatorname{Imt}=-i \beta_{*} / 3$, or even a contour that has a diagonal piece. For vacuum correlators 
many such contours would give the same CFT correlation functions as computed from the AdS/CFT prescription in [6, 7]. However, the interpretation of these other contours both

in bulk terms and in CFT terms is not clear. (Also see 34] for similar remarks.) It would be interesting to either eliminate or understand the physical interpretation of these other contours.

In principle, one could directly compute CFT correlators in the presence of the nonequilibrium density matrix corresponding to an infalling wavepacket. These are expected to be finite and well defined, and should carry information concerning how string theory resolves the inner horizon instability of rotating AdS black holes.

\section{Acknowledgments}

We would like to thank V. Jejjala, D. Mateos, A. Naqvi, and J. Simon for extensive discussions. We thank S. Ross for valuable discussions and collaboration during the early stages of this work. V.B. thanks Aruna Beatrix Balasubramanian who was born during the writing of this paper and assisted in many an editing session. Work on this project was supported by the DOE under cooperative research agreement DE-FG02-95ER40893, by the NSF under grant PHY-0331728 and by an NSF Focused Research Grant DMS0139799 for "The Geometry of Superstrings".

[1] P. R. Brady and C. M. Chambers, "Nonlinear instability of Kerr type Cauchy horizons," Phys. Rev. D 51, 4177 (1995) arXiv:gr-qc/9501025.

[2] R. Penrose, in Batelle Rencontres, eds. C. de Witt and J. Wheeler (W.A. Benjamin, New York, 1968), p. 222. R. A. Matzner, N. Zamorano and V. D. Sanberg, "Instability of the Cauchy horizon of Reissner-Nordström black holes,” Phys. Rev. D 19, 2821 (1979); S. Chandrasekhar and J. Hartle, "On crossing the Cauchy horizon of a Reissner-Nordström black hole," Proc. Roy. Soc. Lond. A384, 301 (1982).

[3] M. Dafermos, "The interior of charged black holes and the problem of uniqueness in general relativity," arXiv:gr-qc/0307013; M. Dafermos and I. Rodnianski, "A proof of Price's law for the collapse of a self-gravitating scalar field," arXiv:gr-qc/0309115. M. Dafermos, "Price's law, 
mass inflation, and strong cosmic censorship," arXiv:gr-qc/0401121,

[4] R. Penrose, "Gravitational Collapse: The Role Of General Relativity," Riv. Nuovo Cim. 1 (1969) 252 [Gen. Rel. Grav. 34 (2002) 1141]; R. Penrose, "Singularities and time-asymmetry," in General Relativity, an Einstein Centenary Survey, eds. S.W. Hawking and W. Israel (Cambridge University Press, 1979).

[5] S. Hemming, E. Keski-Vakkuri and P. Kraus, "Strings in the extended BTZ spacetime," JHEP 0210, 006 (2002) arXiv:hep-th/0208003.

[6] P. Kraus, H. Ooguri and S. Shenker, "Inside the horizon with AdS/CFT," Phys. Rev. D 67, 124022 (2003) arXiv:hep-th/0212277.

[7] T. S. Levi and S. F. Ross, "Holography beyond the horizon and cosmic censorship," Phys. Rev. D 68, 044005 (2003) arXiv:hep-th/0304150.

[8] L. Fidkowski, V. Hubeny, M. Kleban and S. Shenker, "The black hole singularity in AdS/CFT," JHEP 0402, 014 (2004) arXiv:hep-th/0306170.

[9] J. Kaplan, "Extracting data from behind horizons with the AdS/CFT correspondence," arXiv:hep-th/0402066

[10] V. Balasubramanian, A. Naqvi and J. Simon, "A multi-boundary AdS orbifold and DLCQ holography: A universal holographic description of extremal black hole horizons," arXiv:hep-th/0311237

[11] M. Banados, C. Teitelboim and J. Zanelli, "The Black Hole In Three-Dimensional SpaceTime," Phys. Rev. Lett. 69, 1849 (1992) arXiv:hep-th/9204099.

[12] M. Banados, M. Henneaux, C. Teitelboim and J. Zanelli, "Geometry of the (2+1) black hole," Phys. Rev. D 48, 1506 (1993) arXiv:gr-qc/9302012.

[13] V. Husain, "Radiation Collapse And Gravitational Waves In Three-Dimensions," Phys. Rev. D 50, 2361 (1994) arXiv:gr-qc/9404047. J. S. F. Chan, K. C. K. Chan and R. B. Mann, "Interior Structure Of A Charged Spinning Black Hole In (2+1)-Dimensions," Phys. Rev. D 54, 1535 (1996) arXiv:gr-qc/9406049.

[14] E. Keski-Vakkuri, "Bulk and boundary dynamics in BTZ black holes," Phys. Rev. D 59, 104001 (1999) arXiv:hep-th/9808037.

[15] M. Abramowitz and I. Stegun (Ed.), "Handbook of mathematical functions, with formulas, graphs, and mathematical tables," Washington D.C.: U.S. Govt. Print. Off. (1964).

[16] N. D. Birrell and P. C. W. Davies, "Quantum Fields In Curved Space," New York: Cambridge 
Univ. Press, (1982).

[17] G. T. Horowitz and V. E. Hubeny, "Quasinormal modes of AdS black holes and the approach to thermal equilibrium," Phys. Rev. D 62, 024027 (2000) arXiv:hep-th/9909056; V. Cardoso and J. P. S. Lemos, "Scalar, electromagnetic and Weyl perturbations of BTZ black holes: Quasi normal modes," Phys. Rev. D 63, 124015 (2001) arXiv:gr-qc/0101052; D. Birmingham, "Choptuik scaling and quasinormal modes in the AdS/CFT correspondence," Phys. Rev. D 64, 064024 (2001) arXiv:hep-th/0101194; V. Cardoso, R. Konoplya and J. P. S. Lemos, "Quasinormal frequencies of Schwarzschild black holes in anti-de Sitter spacetimes: A complete study on the asymptotic behavior," Phys. Rev. D 68, 044024 (2003) arXiv:gr-qc/0305037; V. Cardoso, J. Natario and R. Schiappa, "Asymptotic quasinormal frequencies for black holes in non-asymptotically flat spacetimes," arXiv:hep-th/0403132.

[18] W. G. Unruh, "Notes On Black Hole Evaporation," Phys. Rev. D 14, 870 (1976).

[19] D. G. Boulware, "Quantum Field Theory In Schwarzschild And Rindler Spaces," Phys. Rev. D 11, 1404 (1975).

[20] J. M. Maldacena, "The large N limit of superconformal field theories and supergravity," Adv. Theor. Math. Phys. 2, 231 (1998) [Int. J. Theor. Phys. 38, 1113 (1999)] arXiv:hep-th/9711200.

[21] S. S. Gubser, I. R. Klebanov and A. M. Polyakov, "Gauge theory correlators from non-critical string theory," Phys. Lett. B 428, 105 (1998) arXiv:hep-th/9802109.

[22] E. Witten, "Anti-de Sitter space and holography," Adv. Theor. Math. Phys. 2, 253 (1998) arXiv:hep-th/9802150.

[23] G. T. Horowitz and S. F. Ross, "Possible resolution of black hole singularities from large N gauge theory," JHEP 9804, 015 (1998) arXiv:hep-th/9803085.

[24] J. Louko and D. Marolf, "Single-exterior black holes and the AdS-CFT conjecture," Phys. Rev. D 59, 066002 (1999) arXiv:hep-th/9808081.

[25] V. Balasubramanian and S. F. Ross, "Holographic particle detection," Phys. Rev. D 61, 044007 (2000) arXiv:hep-th/9906226;

[26] J. Louko, D. Marolf and S. F. Ross, "On geodesic propagators and black hole holography," Phys. Rev. D 62 (2000) 044041 arXiv:hep-th/0002111.

[27] J. M. Maldacena, "Eternal black holes in Anti-de-Sitter," JHEP 0304, 021 (2003) arXiv:hep-th/0106112. 
[28] V. E. Hubeny, "Precursors see inside black holes," Int. J. Mod. Phys. D 12, 1693 (2003) arXiv:hep-th/0208047.

[29] V. Balasubramanian, P. Kraus and A. E. Lawrence, "Bulk vs. boundary dynamics in anti-de Sitter spacetime," Phys. Rev. D 59, 046003 (1999) arXiv:hep-th/9805171.

[30] V. Balasubramanian, P. Kraus, A. E. Lawrence and S. P. Trivedi, "Holographic probes of anti-de Sitter space-times," Phys. Rev. D 59, 104021 (1999) arXiv:hep-th/9808017.

[31] P. Kraus and F. Wilczek, "Selfinteraction correction to black hole radiance," Nucl. Phys. B 433, 403 (1995) arXiv:gr-qc/9408003; V. Balasubramanian and H. Verlinde, "BackReaction and Complementarity in 1+1 Dilaton Gravity," Nucl. Phys. B 464, 213 (1996) arXiv:hep-th/9512148; $\quad$ E. Keski-Vakkuri and P. Kraus, "Microcanonical D-branes and back reaction," Nucl. Phys. B 491, 249 (1997) arXiv:hep-th/9610045.

[32] G. T. Horowitz and D. Marolf, "A new approach to string cosmology," JHEP 9807, 014 (1998) arXiv:hep-th/9805207.

[33] E. Calzetta and B. L. Hu, "Nonequilibrium Quantum Fields: Closed Time Path Effective Action, Wigner Function And Boltzmann Equation," Phys. Rev. D 37, 2878 (1988). P. A. Henning, "On The Treatment Of Initial Correlations In Quantum Field Theory Of Nonequilibrium States," Nucl. Phys. B 337, 547 (1990).

[34] C. P. Herzog and D. T. Son, "Schwinger-Keldysh propagators from AdS/CFT correspondence," JHEP 0303, 046 (2003) arXiv:hep-th/0212072. 